\title{
Mollusca from a species-rich deep-water Leptometra community in the Alboran Sea
}

\author{
Serge Gofas ${ }^{1}$, Carmen Salas ${ }^{1}$, José Luis Rueda ${ }^{2}$, Jesús Canoura ${ }^{3}$, Carlos Farias ${ }^{3}$, Juan Gil ${ }^{3}$ \\ ${ }^{1}$ Depto. Biología Animal, Facultad de Ciencias, Universidad de Málaga, E-29071 Málaga, Spain. E-mail: sgofas@uma.es \\ ${ }^{2}$ Centro Oceanográfico de Málaga, Instituto Español de Oceanografía, Puerto Pesquero s/n, E-29640 Fuengirola, Spain. \\ E-mail: jose.rueda@ma.ieo.es \\ ${ }^{3}$ Centro Oceanográfico de Cádiz, Instituto Español de Oceanografía, Apdo. 2609, Puerto Pesquero, Muelle de Levante s/n, \\ E-11006 Cádiz, Spain. E-mail: carlos.farias@cd.ieo.es
}

\begin{abstract}
Summary: An exceptional species richness for Mollusca was found on Avempace bank (349-365 m), Djibouti group, Alboran Sea, where the most abundant species was the crinoid Leptometra phalangium. A sample of sediment sieved on a 0.5 $\mathrm{mm}$ mesh yielded 156 species of molluscs (83 live-taken, 1772 specimens) with a high Shannon-Wiener diversity index $\left(\mathrm{H}^{\prime}{ }_{(\mathrm{log} 2)}=3.60\right)$. The dominant mollusc was Limopsis aurita and the six most common species accounted for $77 \%$ of the specimens. On the other hand, 42 species were represented by only one or two specimens. Two species are described as new, three more are first findings in the Mediterranean and two are first findings in Iberian waters. One-third of the species have not been reported from the neighbouring, well-explored Alboran Island platform. There is a considerable balance between the trophic groups, denoting a stable and structured community. Most of the species have an extensive Atlantic range, and most have a planktonic larval stage. Therefore, it can be hypothesized that the fauna benefits from a diverse influx of larvae carried in by the more superficial incoming Atlantic current. Because of its exceptional richness, this type of bathyal community with Leptometra and Limopsis should be considered a high priority for habitat conservation in the Mediterranean deep sea.
\end{abstract}

Keywords: species diversity; taxonomy; dominant species; trophic structure; geographical distribution; bathyal zone; Leptometra phalangium; marine molluscs.

\section{Moluscos de una comunidad profunda de Leptometra rica en especies en el mar de Alborán}

Resumen: Se encontró una excepcional riqueza específica de moluscos en el banco Avempace (349-365 m), grupo de Djibouti, mar de Alborán, donde la especie más abundante fue el crinoideo Leptometra phalangium. En una muestra de sedimento tamizada sobre una malla de $0.5 \mathrm{~mm}$ se hallaron 156 especies de moluscos ( 83 con ejemplares vivos) y 1772 ejemplares, con un alto valor del índice de diversidad Shannon-Wiener $\left(\mathrm{H}^{\prime}\left({ }^{\prime}=3.60\right)\right.$. El molusco dominante era Limopsis aurita y las seis especies más comunes eran el 77\% de los ejemplares. Por otro lado, 42 especies estuvieron representadas únicamente por uno o dos ejemplares. Dos especies se describen como nuevas; tres más son primera cita para el Mediterráneo y dos son primera cita en aguas ibéricas. Un tercio de las especies no están citadas en la plataforma de la vecina isla de Alborán, por su parte bastante estudiada. La representación de los distintos grupos tróficos es equilibrada, denotando una comunidad estable y estructurada. La mayoría de las especies tienen una amplia distribución en el Atlántico y una fase larvaria planctónica. Por lo tanto se supone que la fauna se beneficia de un aporte diverso de larvas por la corriente superficial que entra desde el Atlántico. Por su excepcional riqueza, este tipo de comunidad batial con Leptometra y Limopsis merece considerarse prioritaria para la conservación de hábitats profundos del mar Mediterráneo.

Palabras clave: diversidad especifica; taxonomía; especies dominantes; estructura trófica; distribución geográfica; zona batial; Leptometra phalangium; moluscos marinos.

Citation/Como citar este artículo: Gofas S., Salas C., Rueda J.L., Canoura J., Farias C., Gil J. 2014. Mollusca from a species-rich deep-water Leptometra community in the Alboran Sea. Sci. Mar. 78(4): 537-553. doi: http://dx.doi.org/10.3989/ scimar.04097.27A

LSID http://zoobank.org/E6E11F79-E4F6-4420-9519-2D8F3E8DBE98

Editor: M. Ramón.

Received: May 20, 2014. Accepted: October 14, 2014. Published: November 27, 2014.

Copyright: @ 2014 CSIC. This is an open-access article distributed under the Creative Commons Attribution-Non Commercial Lisence (by-nc) Spain 3.0. 


\section{INTRODUCTION}

The Mediterranean Sea is considered as a marine biodiversity hot spot (Coll et al. 2010) and the western Mediterranean displays the highest values of species richness, likely owing to the communication with the Atlantic and a wide range of physicochemical conditions (UNEP-MAP-RAC/SPA 2010a). The proximity to the strait of Gibraltar, together with its higher productivity, has highlighted the importance of the Alboran basin as one of the main biodiversity hotspots of the Mediterranean Sea (Rueda et al. 2009, Coll et al. 2010).

The Alboran Sea contains a wide variety of ecologically interesting seafloor structures, such as submarine canyons of varying size (Baro et al. 2012), carbonate mounds (Lo Iacono et al. 2008, Pardo et al. 2011), submarine structures caused by fluid emissions such as pockmarks and mud volcanoes (Somoza et al. 2012, Gennari et al. 2013), and seamounts and banks of different heights and geologic origins (Palomino et al. 2011). The diverse seafloor morphology and the location and peculiar hydrodynamic features of the Alboran Sea, with the mixing of Atlantic and Mediterranean waters, promote a wide diversity of habitats and associated biota that have been little studied in specific bathyal areas, especially at a microfaunal level (Salas 1996).

Biogeographically, seamounts and submarine banks function as islands separated by great depths (Samadi et al. 2007). Consequently, they may serve as isolated refuges for many species, including relict populations of species that have disappeared from other areas. A complete and detailed map of all Mediterranean seamounts is not yet available. Most investigations of Mediterranean seamounts have been geological, while biological studies have been relatively scarce (Danovaro et al. 2010) and only recently have research expeditions started to explore the seamounts in the Alboran basin (Hebbeln et al. 2009, this study).

The marine benthic communities of the outer shelf, slope and offshore seamounts or banks of the Alboran Sea are currently being given increasing attention, particularly because the pressure of demersal fishing has become particularly important at this depth range (IEO-MAGRAMA 2012). This triggers a need to identify conservation priorities in the offshore areas. In this context, the project DEEPER ("Desarrollo de Estudios de Ecosistemas de Profundidad bajo un Enfoque interdisciplinaR"; chief scientist, Juan Gil Herrera) was set up by the Instituto Español de Oceanografía between 2006 and 2009, with the objective of assessing deep-sea ecosystems in the Gulf of Cadiz and the Alboran Sea, especially in areas where fisheries exist or are likely to exist in the near future. In a multidisciplinary approach, the project investigated the key geological features characterizing geohabitats in potential trawling grounds, and also aimed to identify the deep sensitive habitats which could be threatened and need to be protected. The particular objective of the DEEPER 04/09 cruise was to study several banks of the Alboran Sea, searching for geohabitats with hydrothermal influence and the occurrence of live or subfossil deep-water corals, and analysing the subfos- sil and living benthic fauna for possible indicators of environmental status.

Mollusca are one of the major groups of the benthos worldwide, making up to $15 \%-25 \%$ of the benthic macrofauna (Appeltans et al. 2012). They have a quite complete record allowing comparisons with neighbouring areas and are considered to be an 'appropriate indicator group' for rapid assessment of diversity inhabiting a particular area (Bedulli et al. 2002, Gladstone 2002, Smith 2005). Moreover, information on molluscs of the Alboran Sea is generally more detailed for infralittoral and circalittoral habitats (Gofas et al. 2011) than for bathyal ones. Therefore the aims of the present study were to analyse (1) the composition and structure and (2) the biogeographical affinity and feeding guilds of the molluscan assemblage of a seamount of the Alboran Sea.

\section{STUDY AREA}

The studied bank is situated on the Motril (or Djibouti) Marginal Plateau, an extensive structural feature which extends around 800-900 m depth on the margin of the southern Iberian Peninsula facing Málaga and Adra (Fig. 1; see also Palomino et al. 2011, Figs 1 and 3 ), with a practically flat seafloor. The seafloor of the plateau is interrupted by the presence of several banks (Algarrobo or Avempace, Djibouti or Herradura, Villede-Djibouti or El Idrissi), mostly with a cone-truncated shape and a rather flat summital area. There is considerable confusion regarding the toponymy of the seafloor features in the Alboran Sea, many of which bear alternative names (UNEP-MAP-RAC/SPA 2010b). Regarding the Motril Marginal Plateau, only the easternmost bank is recorded in the GEBCO undersea feature names gazeteer <http://www.ngdc.noaa.gov/ gazetteer/> under the name "Ville de Djibouti Bank".

Algarrobo Bank (also known as Avempace) is situated to the northwest of the group, culminating at 250 $m$ depth. It covers an area of $83 \mathrm{~km}^{2}$ and is surrounded by steep slopes reaching down to the plateau at nearly $800 \mathrm{~m}$ depth (Palomino et al. 2011); it is therefore here considered a "bank" rather than a "seamount", following IHO guidelines which define a "seamount" as elevated more than $1000 \mathrm{~m}$ above the seafloor (IHO 2013). The summit of Algarrobo Bank is characterized by the occurrence of several mounds and ridges, and is covered by muddy-sandy sediments containing a large fraction of planktonic foraminiferal tests (Gil et al. 2009, García-Muñoz et al. 2012).

The water circulation in the Alboran Sea is known to be strongly influenced by a layer of surface water of Atlantic origin. Three main water masses in the Alboran Sea affect the area of Algarrobo Bank (Parrilla et al. 1986, Sardà et al. 2004, Millot 2009, 2013): the Atlantic water, flowing eastwards from the Strait of Gibraltar between the surface and a depth of $c a$. 150-200 m; the Levantine Intermediate Water, which is formed in the eastern Mediterranean and circulates westwards between approximately 200 and $600 \mathrm{~m}$; and the Deep Western Mediterranean water, originating in the Gulf of Lion, filling the deepest part of the basin and also flowing westward. 


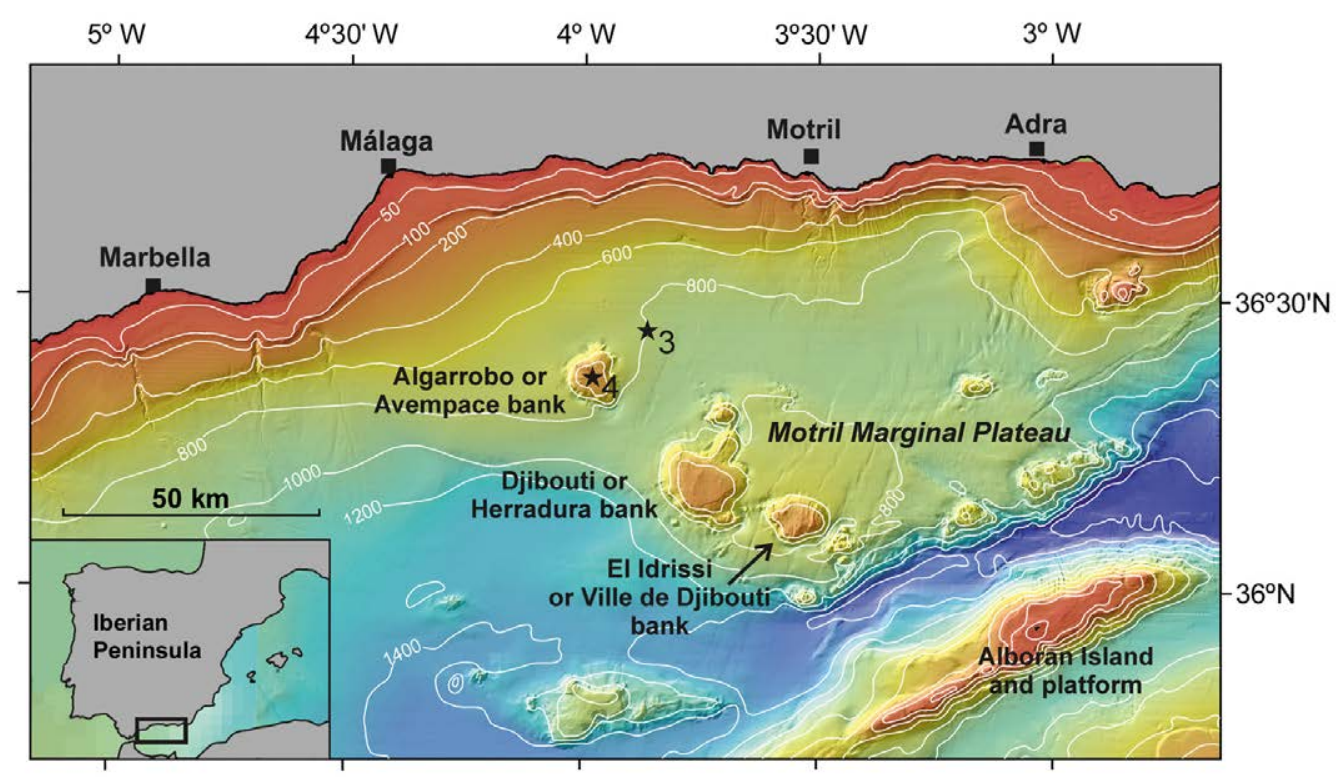

Fig. 1. - Bathymetric map of the study area, showing the main morphological features. The stars indicate the position of the conductivitytemperature-depth stations of Figure 2 (isobaths in metres; adapted from Secretaría General de Pesca Marítima, 2004).

\section{MATERIALS AND METHODS}

The DEEPER 04/09 cruise was carried out on board $\mathrm{R} / \mathrm{V}$ Cornide de Saavedra of the Instituto Español de Oceanografía, from March 31 to April 6, 2009 and performed a sampling consisting of 13 rock dredge hauls, 35 shipek grabs, 7 beam trawl hauls and 4 otter trawl hauls over the three banks of the Djibouti group. Fifteen conductivity-temperature-depth (CTD) profiles were also taken between the edge of the mainland shelf and the surroundings of the banks, one of them situated over Algarrobo Bank.

An exceptional species richness of molluscs was only registered in one particular haul collected with the beam trawl on Algarrobo Bank (BT04), the description of which is the object of this paper. Due to the vulnerable status of the studied habitat, no haul replicate was made. Beam trawl BT04 was operated on the summital area of Algarrobo Bank on April 4, 2009, with the following coordinates:

Start: $36^{\circ} 21.08^{\prime} \mathrm{N}, 03^{\circ} 58.58^{\prime} \mathrm{E}, 349 \mathrm{~m}$ depth End: $36^{\circ} 21.06^{\prime} \mathrm{N}, 03^{\circ} 58.07^{\prime} \mathrm{E}, 365 \mathrm{~m}$ depth

The beam trawl had an opening of $2.4 \mathrm{~m}$ and a codend net of ca. $10 \mathrm{~mm}$ mesh and was towed at a speed of one knot for 12 minutes after reaching the bottom. The sampled area was therefore ca. $889 \mathrm{~m}^{2}$. The catch was immediately discharged on deck into large buckets with sea water, and washed through a series of sieves of 10, 5, 2, 1 and $0.5 \mathrm{~mm}$ mesh size. Despite the large size of the cod-end mesh, it retained a large amount of sediment and fine fraction due to tension and clogging of the net, so it was possible to obtain a representative sampling of the smaller macrofauna.

All the macrofauna larger than $10 \mathrm{~mm}$ was sorted to species level on board, the catch was counted and weighed for each species, and most material of the common species was discarded. A sample of ca. 10 litres of the sediment fraction less than $10 \mathrm{~mm}$ was preserved in $96^{\circ}$ alcohol for later sorting in the laboratory, and provided most of the material of small species studied herein.

Each size fraction (5-10, 2-5, 1-2 and 0.5-1 mm) was sorted separately under the stereomicroscope. Specimens were preserved in $70 \%$ alcohol in the case of non-shelled invertebrates and for a representation of shelled Mollusca; otherwise, most of the shelled Mollusca were dried, a process which ensures a better long-term conservation of the specimens than the wet collection. The species level identification of the Mollusca essentially follows Gofas et al. (2011), an identification guide which was drawn up at the time and in which some specimens of the present material were included. The solenogastres from this and other deepsea cruises off the southern Iberian Peninsula will be studied in detail elsewhere (Pedrouzo et al. 2014) with the description an additional species Alexandromenia avempacensis Pedrouzo and Cobo, 2014, not included in the present species counts. All taxonomic names were checked against the World Register of Marine Species (WoRMS editorial board 2014).

Live-taken specimens and shells were counted separately and are listed in Table 1. Photographs were taken for species of interest using a Nikon DXM camera mounted on a stereomicroscope; for the smaller specimens several views focused on different planes and were assembled with the Automontage software, combining the best-focused parts of each view into a single image.

The identified species were assigned to trophic groups, largely following the guidelines in Rueda et al. (2009), considering all the following categories which are represented in the aphotic zone: (1) carnivores (CA), feeding on other mobile organisms, such as molluscs and polychaetes; (2) scavengers (SC), feeding on remains of dead organisms; (3) deposit feeders (DE), feeding on organic particles contained in the sediment; (4) ectoparasites and specialized carnivores (EC), feeding on much larger organisms on which they live during their 
Table 1. - Shipboard faunal list of haul BT04 with notes on biomass (Weight, g) and abundance ( $\mathrm{N}$, number of individuals or colonies) of each species. These figures relate to a sampled area of $889 \mathrm{~m}^{2}$.

\begin{tabular}{|c|c|c|}
\hline & Weight & $\mathrm{N}$ \\
\hline \multicolumn{3}{|l|}{ Fish } \\
\hline Bathysolea profundicola (Vaillant, 1888) & 124 & 8 \\
\hline Gadiculus argenteus Guichenot, 1850 & 3 & 12 \\
\hline Galeus melastomus Rafinesque, 1810 & 3 & 1 \\
\hline Helicolenus dactylopterus (Delaroche, 1809) & 406 & 3 \\
\hline Hoplostethus mediterraneus Cuvier, 1829 & 51 & 3 \\
\hline Myctophum punctatum Rafinesque, 1810 & 10 & 3 \\
\hline Phycis blennoides (Brünnich, 1768) & 1 & 1 \\
\hline Symphurus nigrescens Rafinesque, 1810 & 14 & 3 \\
\hline Synchiropus phaeton (Günther, 1861) & 5 & 2 \\
\hline \multicolumn{3}{|l|}{ Decapods } \\
\hline Anapagurus laevis (Bell, 1846) & 1 & 1 \\
\hline Athanas nitescens (Leach, 1813) & 1 & 1 \\
\hline Cymonomus granulatus (Norman, 1873) & 1 & 4 \\
\hline Ebalia nux A. Milne-Edwards, 1883 & 45 & 100 \\
\hline Ergasticus clouei A. Milne-Edwards, 1882 & 9 & 3 \\
\hline Eurynome spinosa Hailstone, 1835 & 1 & 1 \\
\hline Lophogaster typicus M. Sars, 1857 & 4 & 27 \\
\hline Macropipus tuberculatus (Roux, 1830) & 4 & 1 \\
\hline Monodaeus couchii (Couch, 1851) & 1 & 2 \\
\hline Munida intermedia A. Milne-Edw. and Bouvier, 1899 & 2 & 1 \\
\hline Munida iris A. Milne-Edwards, 1880 & 2 & 13 \\
\hline Parapenaeus longirostris (Lucas, 1846) & 56 & 7 \\
\hline Periclimenes granulatus Holthuis, 1950 & 2 & 3 \\
\hline Philocheras echinulatus (M. Sars, 1862) & 1 & 3 \\
\hline Plesionika antigai Zariquiey Alvarez, 1955 & 74 & 47 \\
\hline Plesionika giglioli (Senna, 1902) & 1 & 1 \\
\hline Plesionika heterocarpus (A. Costa, 1871) & 2 & 3 \\
\hline Processa nouveli Al-Adhub and Williamson, 1975 & 2 & 9 \\
\hline Solenocera membranacea (Risso, 1816) & 1 & 1 \\
\hline \multicolumn{3}{|l|}{ Molluscs } \\
\hline Abra longicallus (Scacchi, 1835) & 2 & 3 \\
\hline Antalis panorma (Chenu, 1843) & 2 & 8 \\
\hline Heliacus fallaciosus (Tiberi, 1872) & 2 & 1 \\
\hline Limopsis aurita (Brocchi, 1814) & 53 & 265 \\
\hline Lunatia fusca (Blainville, 1825) & 3 & 7 \\
\hline Philine quadripartita Ascanius, 1772 & 2 & 1 \\
\hline Xenophora crispa (König, 1825) & 7 & 2 \\
\hline \multicolumn{3}{|l|}{ Echinoderms } \\
\hline Astropecten sp. & 5 & 2 \\
\hline Leptometra phalangium (J. Müller, 1841) & 815 & 815 \\
\hline Parastichopus regalis (Cuvier, 1817) & 265 & 2 \\
\hline \multicolumn{3}{|l|}{ Others } \\
\hline Aphrodite sp. & 3 & 1 \\
\hline Hyalinoecia tubicola (O.F. Müller, 1776) & 16 & 20 \\
\hline Kophobelemnon stelliferum (O.F. Müller, 1776) & 13 & 11 \\
\hline Thenea muricata (Bowerbank, 1858) & 67 & 35 \\
\hline
\end{tabular}

life cycle; and (5) filter feeders (FI), capturing the seston particles with their gills and/or with mucous strings.

Information on the distribution patterns of molluscs outside the Alboran Sea is given by Bouchet and Warén (1980, 1985, 1986, 1993), Gofas et al. (2011), and Hernández et al. (2011), all of which took into account many area-specific sources. We distinguished the following units: (0) Alboran Sea, for all the species found in this study; (1) Mediterranean Sea (ME), excluding from this category those species that are not reported east of the Alboran Sea; (2) Ibero-Moroccan Gulf, including the southern coasts of Portugal and the Atlantic coasts of Andalusia (southwestern Spain) and Morocco; (3) western Europe, from Portugal to the southern coasts of the United Kingdom; (4) northern Europe, from the southern coasts of the United Kingdom to Scandinavia; (5) the Canary Islands and/or Madeira and (6) western Africa, from Mauritania to tropical western African coasts. The dataset was then analysed using presence/absence data and a Bray-Cur- tis similarity index in order to characterize groups of species sharing similar distributional ranges; a few species (poorly known, or restricted to the Alboran Sea) for which no geographic data are presented in Table 2 were not considered in this analysis.

Larval development was scored for each species as planktonic, planktotrophic or direct, according to the characters of the larval shell (Jablonski and Lutz 1980). In gastropods, a planktotrophic development is denoted by a multispiral protoconch (usually over two whorls, sometimes up to four) comprising a protoconch I and protoconch II, and a clear protoconch/teleoconch separation; a short planktonic but non-planktotrophic development (mostly the Vetigastropoda) is denoted by a very small paucispiral protoconch clearly distinct from the teleoconch; direct development is denoted by a large paucispiral protoconch, usually with an indistinct protoconch/teleoconch transition. In bivalves, planktotrophic development is indicated by a large prodissoconch with distinct prodissoconch I and prodissoconch II; in most species development is achieved through a short-lived planktonic stage corresponding to a simple prodissoconch; rarely, a direct development is denoted by a large prodissoconch without a distinct prodissoconch I/II.

A dominance index (percentage of the species in the total live-collected specimens) was calculated for species and the Shannon-Wiener diversity index (Krebs 1989) and Evenness index (Pielou 1969) were also calculated using the Primer 6 software.

\section{RESULTS}

\section{Oceanographic setting}

The CTD profiles obtained over and near Algarrobo Bank (Fig. 2) clearly indicate that the water mass covering the summit of the bank is the Levantine Intermediate Water. The decrease in salinity and increase in temperature triggered by mixing with the inflowing Atlantic water is not appreciable below $250 \mathrm{~m}$ and therefore one hundred metres above the summit of the bank.

\section{Macrofauna}

The most abundant species was the crinoid Leptometra phalangium, represented in the sample by 815 individuals. Other important species of the benthic macrofauna sorted on board (Table 1) were the bivalve Limopsis aurita, the crab Ebalia nux, the shrimps Plesionika antigai and Lophogaster typicus, the poriferan Thenea muricata and the polychaete Hyalinoecia tubicola.

\section{The molluscan assemblage}

The sample below the $10 \mathrm{~mm}$ size fraction yielded 156 species of molluscs (Table 2), of which 83 were represented by at least one live specimen (total 1772 live-taken specimens and 1656 shells) in the fraction sorted down to $0.5 \mathrm{~mm}$. Considering only the live-collected molluscs, the assemblages feature a high value 

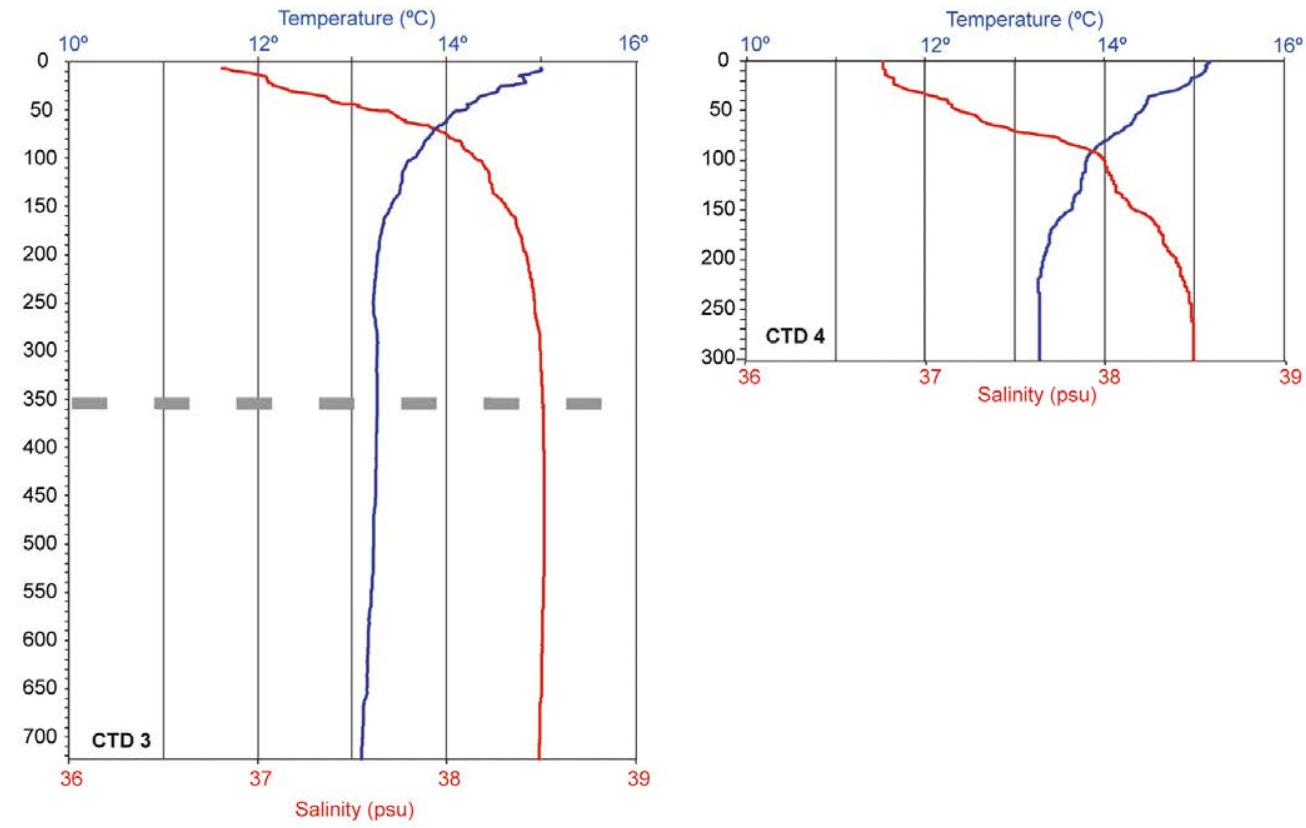

Fig. 2. - Conductivity-temperature-depth (CTD) profiles obtained over Algarrobo Bank and nearby (see Fig. 1). The dashed line on CTD 3 indicates the approximate depth of haul BT04 made at the adjacent Algarrobo Bank, over which the CTD 4 was taken. Superficial layers with salinity less than $37.5 \mathrm{psu}$ are influenced by the inflow of Atlantic origin. Below ca. $250 \mathrm{~m}$ depth, the water temperature stabilizes slightly over $13^{\circ} \mathrm{C}$ and salinity near 38.5 psu, values indicating the westward flowing Levantine Intermediate Water.

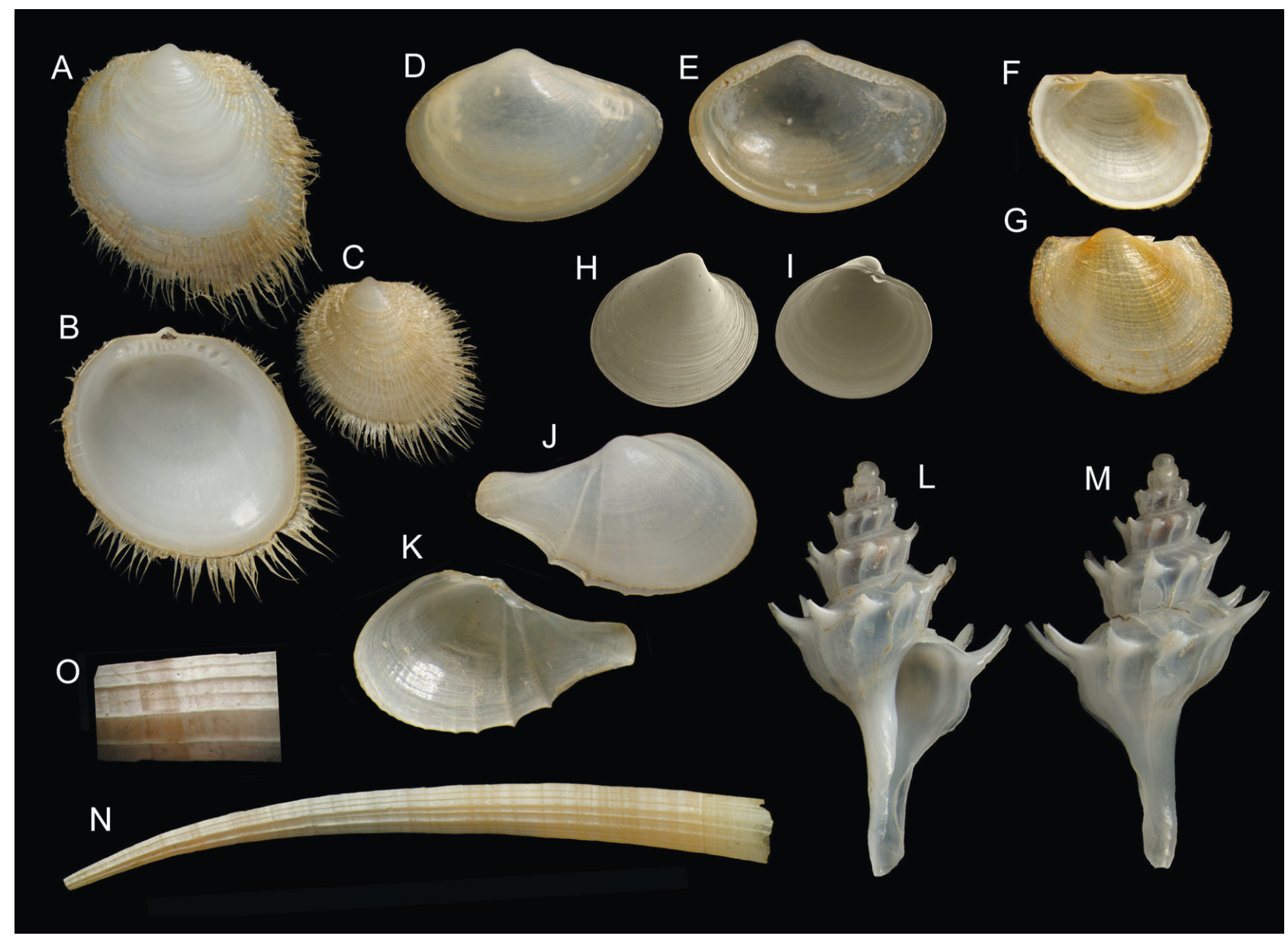

Fig. 3. - Top-dominant and characteristic species from the sediment fraction of haul BT04. A-C: Limopsis aurita (heights $10.0 \mathrm{~mm}$ and 6.4 $\mathrm{mm}$ ). D-E: Yoldiella philippiana (length $5.0 \mathrm{~mm}$ ). F-G: Bathyarca pectunculoides (lengths 3.4 and $2.8 \mathrm{~mm}$ ). H-I: Kelliella miliaris (scanning electron micrograph; lengths 1.8 and $1.6 \mathrm{~mm}$ ); J-K: Cardiomya costellata (length $5.6 \mathrm{~mm}$ ). L-M: Pagodula echinata (height $8.0 \mathrm{~mm}$ ), N-O: Antalis panorma (length $39.0 \mathrm{~mm}$ ) and detail of sculpture. 
Table 2. - Faunistic list of molluscs found in haul BT04 of the DEEPER 0409 expedition. Live, number of live-collected specimens; sh, number of shells or valves. Alb, presence in Alboran platform denoted by + (according to Peñas et al. 2006 and unpublished results from INDEMARES Alboran). Codes for trophic groups (Tr.): CA, carnivores; SC, scavengers; DE, deposit feeders; EC, ectoparasites and specialized carnivores; FI, filter feeders. Codes for biogeographical distribution: ME, Mediterranean Sea; IB, Ibero-Moroccan Gulf; WE, western Europe; NE, northern Europe; CN, Canary Islands and Madeira; WA, western Africa). Codes for inferred type of larval developments (larv.): SP, short planktonic; PK, planktotrophic; DI, direct. Species denoted by $\dagger$ are suspected to belong to cold-water thanatocoenoses.

\begin{tabular}{|c|c|c|c|c|c|c|c|c|c|c|c|c|}
\hline Species & Authority & live & sh. & Alb & Tr. & $\mathrm{ME}$ & IB & WE & $\mathrm{NE}$ & $\mathrm{CN}$ & WA & larv \\
\hline \multicolumn{13}{|l|}{ SOLENOGASTRES } \\
\hline Neomenia carinata & Tullberg, 1875 & 5 & & + & $\mathrm{EC}$ & + & + & + & + & & & SP \\
\hline Unciherpia hirsuta & $\begin{array}{l}\text { García-Alvarez, Urgorri and } \\
\text { Salvini-Plawen, } 2001\end{array}$ & 5 & & & $\overline{\mathrm{EC}}$ & & & + & & & & SP \\
\hline \multicolumn{13}{|l|}{ GASTROPODA } \\
\hline Propilidium exiguum & (Thompson, 1844) & & 11 & + & & & + & + & + & + & & SP \\
\hline Iothia fulva & (O.F. Müller, 1776) & & 26 & & & & & & + & & & SP \\
\hline Bogia labronica & (Bogi, 1984) & & 1 & & & + & & & & & & SP \\
\hline Puncturella noachina ${ }^{\dagger}$ & (Linnaeus, 1771) & & 39 & & & & & & + & & & SP \\
\hline Emarginula fissura & (Linnaeus, 1758) & & 3 & + & & + & + & + & + & + & & SP \\
\hline Fissurisepta granulosa & Jeffreys, 1883 & & 2 & & & & & & + & & & SP \\
\hline Anatoma aspera & (Philippi, 1844) & 24 & 17 & + & $\mathrm{DE}$ & + & + & + & + & + & + & $\mathrm{SP}$ \\
\hline Anatoma tenuisculpta & (Seguenza, 1880) & & 13 & + & & & + & + & + & & & SP \\
\hline Addisonia excentrica & (Tiberi, 1855) & & 2 & + & & + & + & + & & + & + & SP \\
\hline Lepetella laterocompressa & (de Rayneval and Ponzi, 1854) & & 7 & + & & + & + & + & + & & & SP \\
\hline Calliostoma granulatum & (Born, 1778) & 2 & & + & $\mathrm{EC}$ & + & + & + & & + & & SP \\
\hline Calumbonella suturalis & (Philippi, 1836) & 5 & & & & & + & + & & & & SP \\
\hline Clelandella miliaris & (Brocchi, 1814) & & 7 & + & & + & + & + & + & & + & SP \\
\hline Danilia tinei & (Calcara, 1839) & 1 & 1 & + & $\mathrm{DE}$ & + & + & + & + & + & & SP \\
\hline Adeuomphalus ammoniformis & (Seguenza, 1876) & 1 & & + & & & & & & & & SP \\
\hline Cirsonella romettensis & (Granata-Grillo, 1877) & 4 & 32 & + & $\mathrm{DE}$ & + & + & + & & & & SP \\
\hline Lissotesta gittenbergeri & (van Aartsen and Bogi, 1988) & & 1 & + & & + & + & & & & & SP \\
\hline Anekes sculpturata & Warén, 1992 & 1 & 1 & & $\mathrm{DE}$ & + & + & + & & & & SP \\
\hline Turritella communis & Risso, 1826 & 2 & & & FI & + & + & + & + & & & SP \\
\hline Strobiligera brychia & (Bouchet and Guillemot, 1978) & & 1 & + & & & + & + & & + & & PK \\
\hline Cerithiella metula & (Lovén, 1846) & & 1 & & & & + & + & + & + & & DI \\
\hline Alvania cimicoides & (Forbes, 1844) & 2 & 18 & + & $\mathrm{DE}$ & + & + & + & + & + & & PK \\
\hline Alvania testae & (Aradas and Maggiore, 1844) & 1 & 4 & + & $\mathrm{DE}$ & + & + & + & + & & & $\mathrm{PK}$ \\
\hline Alvania porcupinae & Gofas and Warén, 1982 & & 29 & & & & + & + & & + & & \\
\hline Alvania zetlandica & (Montagu, 1815) & & 10 & + & & + & + & + & + & + & & PK \\
\hline Talassia dagueneti & (de Folin, 1873) & & 8 & + & & + & + & + & & & & \\
\hline Euspira fusca & (Blainville, 1825) & 9 & $\mathrm{nc}$ & + & $\mathrm{CA}$ & + & + & + & + & & + & PK \\
\hline Protatlanta souleyeti & (Smith E.A., 1888) & & 1 & & & + & + & + & & + & + & $\mathrm{PK}$ \\
\hline Aclis attenuans & Jeffreys, 1883 & & 7 & & & + & & & & & & $\mathrm{PK}$ \\
\hline Aclis gulsonae & (Clark W., 1850) & & 10 & + & & + & + & + & + & & & \\
\hline Epitonium algerianum & (Weinkauff, 1866) & 1 & 14 & + & $\mathrm{EC}$ & + & + & + & & + & + & PK \\
\hline Epitonium celesti & (Aradas, 1854) & 1 & 4 & + & $\mathrm{EC}$ & + & + & + & & + & & $\mathrm{PK}$ \\
\hline Epitonium linctum & (de Boury and Monterosato, 1890) & & 9 & + & & + & + & & & & & $\mathrm{PK}$ \\
\hline Epitonium clathratulum & (Kanmacher, 1798) & & 1 & + & & + & + & + & + & + & & $\mathrm{PK}$ \\
\hline Epitonium hispidulum & (Monterosato, 1874) & 1 & 9 & + & $\mathrm{EC}$ & + & + & + & & + & & $\mathrm{PK}$ \\
\hline Acirsa subdecussata & (Cantraine, 1835) & & 1 & + & & + & + & + & & + & & $\mathrm{PK}$ \\
\hline Opaliopsis atlantis & (Clench and Turner, 1952) & & 2 & + & & + & + & & & + & & $\mathrm{PK}$ \\
\hline Eulima fuscozonata & Bouchet and Warén, 1986 & 1 & 4 & + & $\mathrm{EC}$ & & + & & & + & & \\
\hline Eulima bilineata & Alder, 1848 & 1 & 2 & + & $\mathrm{EC}$ & + & + & + & + & & & \\
\hline Melanella frielei & (Jordan, 1895) & 4 & 5 & + & $\mathrm{EC}$ & + & + & + & + & + & & PK \\
\hline Melanella scarifata & n. sp. & 1 & & & & & & & & & & $\mathrm{PK}$ \\
\hline Melanella petitiana & (Brusina, 1869) & 1 & 1 & + & $\mathrm{EC}$ & + & + & + & & & & $\mathrm{PK}$ \\
\hline Curveulima beneitoi & Peñas and Rolán, 2006 & & 4 & + & & & & & & & & DI \\
\hline Curveulima devians & (Monterosato, 1884) & 11 & 1 & + & $\mathrm{EC}$ & + & & & & & & $\mathrm{PK}$ \\
\hline Trophonopsis barvicensis & (Johnston, 1825) & 14 & 7 & & $\mathrm{CA}$ & & + & + & + & & & DI \\
\hline Pagodula echinata & (Kiener, 1840) & 70 & 15 & & $\mathrm{CA}$ & + & + & + & + & & & DI \\
\hline Mitrella profundi & (Gould, 1840) & & 8 & & & & & & & & & $\mathrm{PK}$ \\
\hline Amphissa acutecostata & (Philippi, 1844) & & 1 & & & + & + & + & + & + & & $\mathrm{PK}$ \\
\hline Pseudobabylonella minima & (Reeve, 1856) & & 8 & + & & & + & & & + & & DI \\
\hline Drilliola loprestiana & (Calcara, 1841) & 6 & 3 & + & $\mathrm{CA}$ & + & + & + & & + & & PK \\
\hline Drilliola emendata & (Monterosato, 1872) & 2 & 5 & & $\mathrm{CA}$ & + & + & & & & & DI \\
\hline Spirotropis monterosatoi & (Locard, 1897) & 23 & 16 & & $\mathrm{CA}$ & & + & & & + & & DI \\
\hline Spirotropis modiolus & (de Cristofori and Jan, 1832) & 7 & 11 & & $\mathrm{CA}$ & + & + & + & + & & & DI \\
\hline Mangelia nuperrima & (Tiberi, 1855) & 6 & 4 & + & $\mathrm{CA}$ & + & + & & & & & $\mathrm{PK}$ \\
\hline Mangelia costata & (Pennant, 1777) & 4 & 3 & + & $\mathrm{CA}$ & + & + & + & + & & & $\mathrm{PK}$ \\
\hline Teretia teres & (Reeve, 1844) & & 5 & + & & + & + & + & + & + & & $\mathrm{PK}$ \\
\hline Raphitoma echinata & (Brocchi, 1814) & 1 & 1 & + & $\mathrm{CA}$ & + & + & + & + & + & & $\mathrm{PK}$ \\
\hline Bela atlantidea & (Knudsen, 1956) & & 2 & & & + & + & & & + & + & $\mathrm{PK}$ \\
\hline Pleurotomella gibbera & Bouchet and Warén, 1980 & 2 & 4 & + & $\mathrm{CA}$ & + & + & & & + & & $\mathrm{PK}$ \\
\hline Gymnobela abyssorum & (Locard, 1897) & 8 & 5 & + & $\mathrm{CA}$ & + & + & + & & & & $\mathrm{PK}$ \\
\hline Spirolaxis clenchi & Jaume and Borro, 1916 & 1 & 2 & & $\mathrm{EC}$ & + & + & & & & & $\mathrm{PK}$ \\
\hline Spirolaxis lamellifer & (Rehder, 1935) & 1 & & & $\mathrm{EC}$ & + & + & & & + & & PK \\
\hline Mathilda coronata & Monterosato, 1875 & & 1 & + & & + & + & & & & & $\mathrm{PK}$ \\
\hline Graphis pruinosa & n. $\mathrm{sp}$. & 1 & 9 & & & & & & & & & DI \\
\hline Graphis gracilis & (Monterosato, 1874) & & 8 & + & & + & + & & & + & & DI \\
\hline Turbonilla magnifica & (Seguenza, 1879) & & 2 & & & & & & & & & PK \\
\hline Turbonilla vaillanti & Dautzenberg and Fischer, 1896 & 1 & & & & & & & & & & $\mathrm{PK}$ \\
\hline Eulimella ventricosa & (Forbes, 1844) & 4 & 7 & + & $\mathrm{EC}$ & + & + & + & + & + & & $\mathrm{PK}$ \\
\hline Tiberia minuscula & (Monterosato, 1880) & 3 & 1 & + & $\mathrm{EC}$ & + & + & + & & & & $\mathrm{PK}$ \\
\hline Chrysallida fenestrata & (Jeffreys, 1848) & & 4 & & & + & + & + & + & + & + & $\mathrm{PK}$ \\
\hline
\end{tabular}




\begin{tabular}{|c|c|c|c|c|c|c|c|c|c|c|c|c|}
\hline Species & Authority & live & sh. & Alb & Tr. & ME & IB & WE & $\mathrm{NE}$ & $\mathrm{CN}$ & WA & larv \\
\hline Chrysallida flexuosa & (Monterosato, 1874) & 7 & 6 & + & $\mathrm{EC}$ & + & + & & & + & & $\mathrm{PK}$ \\
\hline Actaeon monterosatoi & Dautzenberg, 1889 & & 7 & + & & + & + & & & + & & PK \\
\hline Cylichna cylindracea & (Pennant, 1777) & 1 & 3 & + & $\mathrm{CA}$ & + & + & + & + & + & + & $\mathrm{PK}$ \\
\hline Scaphander lignarius & (Linnaeus, 1758) & & 3 & + & & + & + & + & + & + & & $\mathrm{PK}$ \\
\hline Roxania utriculus & (Brocchi, 1814) & 1 & 5 & & $\mathrm{CA}$ & + & + & + & & + & & $\mathrm{PK}$ \\
\hline Ringicula ciommeii & Mariottini, Smriglio and Oliverio, 2000 & & 5 & & & & & & & & & \\
\hline Philine monterosati & Monterosato, 1874 & 2 & 6 & & CA & + & + & + & & & & $\mathrm{PK}$ \\
\hline Philine punctata & (Adams J., 1800) & 8 & 10 & + & $\mathrm{CA}$ & + & + & + & + & & & $\mathrm{PK}$ \\
\hline Philine scabra & (Müller, 1784) & 3 & 12 & & $\mathrm{CA}$ & + & + & + & + & + & & $\mathrm{PK}$ \\
\hline Limacina retroversa ${ }^{\dagger}$ & (Fleming, 1823) & & 28 & + & & & & + & + & & & $\mathrm{PK}$ \\
\hline Clio pyramidata & Linnaeus, 1767 & & 6 & + & & + & + & + & & + & & PK \\
\hline Clio cuspidata & (Bosc, 1802) & & 35 & + & & + & + & + & & + & & PK \\
\hline Peracle reticulata & (d'Orbigny, 1834) & & 1 & + & & & & & & & & $\mathrm{PK}$ \\
\hline Cavolinia inflexa & (Lesueur, 1813) & & 18 & + & & + & + & + & & + & & $\mathrm{PK}$ \\
\hline \multicolumn{13}{|l|}{ BIVALVIA } \\
\hline Nucula tumidula & Malm, 1861 & & 15 & & & & + & + & + & & & SP \\
\hline Ennucula decipiens & (Philippi, 1844) & 8 & 14 & + & $\mathrm{DE}$ & & + & + & & & + & $\mathrm{SP}$ \\
\hline Ennucula aegeensis & (Forbes, 1844) & 2 & 1 & + & $\mathrm{DE}$ & + & + & & & & & SP \\
\hline Ennucula corbuloides ${ }^{\dagger}$ & (Seguenza, 1877) & & 23 & & & & + & + & & & & SP \\
\hline Yoldiella philippiana & (Nyst, 1845) & 275 & 106 & + & $\mathrm{DE}$ & + & + & + & + & & + & SP \\
\hline Yoldiella sp. & & & 3 & & & & & & & & & SP \\
\hline Ledella messanensis & (Jeffreys, 1879) & 1 & 38 & + & $\mathrm{DE}$ & + & + & + & & + & & SP \\
\hline Microgloma pusilla & (Jeffreys, 1870) & & 1 & & & + & + & & & & & SP \\
\hline Bathyarca pectunculoides & (Scacchi, 1835) & 132 & 112 & + & FI & + & + & + & + & + & + & SP \\
\hline Bathyarca philippiana & (Nyst, 1848) & 33 & 19 & + & FI & + & + & + & + & + & & $\mathrm{SP}$ \\
\hline Bathyarca frielei & (Friele, 1877) & & 19 & & & & & & + & & & $\mathrm{SP}$ \\
\hline Asperarca nodulosa & (Müller, 1776) & & 7 & + & & + & + & + & + & + & & $\mathrm{SP}$ \\
\hline Limopsis aurita & (Brocchi, 1814) & 676 & nc & + & FI & + & + & + & + & + & & $\mathrm{SP}$ \\
\hline Limposis minuta & (Philippi, 1836) & & 7 & & & & + & + & & + & & $\mathrm{SP}$ \\
\hline Limopsis friedbergi & Glibert and van de Poel, 1965 & & 1 & & & & & & & & & $\mathrm{SP}$ \\
\hline Pseudamussium clavatum & (Poli, 1795) & & 1 & + & & + & + & + & & + & & PK \\
\hline Pseudamussium sulcatum & (Müller O.F., 1776) & & 1 & + & & + & + & + & + & + & & $\mathrm{PK}$ \\
\hline Delectopecten vitreus & (Gmelin, 1791) & & 1 & + & & + & + & + & + & + & + & $\mathrm{PK}$ \\
\hline Parvamussium fenestratum & (Forbes, 1844) & 28 & 20 & + & FI & + & + & + & + & + & + & $\mathrm{SP}$ \\
\hline Cyclopecten hoskynsi & (Forbes, 1844) & & 9 & + & & + & + & & & & & $\mathrm{SP}$ \\
\hline Similipecten similis & (Laskey, 1811) & 2 & 54 & + & FI & + & + & + & + & + & + & $\mathrm{SP}$ \\
\hline Heteranomia squamula & (Linnaeus, 1758) & & 20 & + & & & + & + & + & + & + & SP \\
\hline Dacrydium ockelmanni & Mattson and Warén, 1977 & & 17 & & & & & + & + & & & SP \\
\hline Crenella pellucida & (Jeffreys, 1859) & 1 & & + & FI & + & + & + & & + & & SP \\
\hline Notolimea crassa & (Forbes, 1844) & 10 & 76 & + & FI & + & + & + & + & & + & SP \\
\hline Limatula gwyni & (Sykes, 1903) & & 15 & + & & + & + & + & + & & + & SP \\
\hline Limatula subovata & (Monterosato, 1875) & 5 & 49 & + & FI & & & & & & & SP \\
\hline Astarte sulcata & (da Costa, 1778) & & 3 & + & & + & + & + & + & & & SP \\
\hline Myrtea spinifera & (Montagu, 1803) & 1 & 1 & + & FI & + & + & + & + & + & + & SP \\
\hline Lucinoma borealis & (Linnaeus, 1767) & & 1 & + & & + & + & + & + & + & & SP \\
\hline Thyasira biplicata & (Philippi, 1836) & & 49 & & & + & + & + & + & & & SP \\
\hline Thyasira granulosa & (Monterosato, 1874) & 2 & 17 & & FI & + & + & + & + & & & SP \\
\hline Thyasira succisa & (Jeffreys, 1876) & 1 & 23 & + & FI & + & + & + & & + & & SP \\
\hline Thyasira subovata & (Jeffreys, 1881) & 2 & 2 & + & FI & + & + & & & & + & $\mathrm{SP}$ \\
\hline Thyasira obsoleta & (Verrill and Bush, 1898) & 1 & & & FI & & + & + & + & & + & SP \\
\hline Thyasira sp. & & & 40 & + & & & & & & & & SP \\
\hline Mendicula ferruginosa & (Forbes, 1844) & 8 & 39 & & FI & + & + & + & + & & + & $\mathrm{SP}$ \\
\hline Axinulus croulinensis & (Jeffreys, 1847) & 6 & 5 & & FI & + & + & + & + & & + & $\mathrm{SP}$ \\
\hline Axinulus eumyarius & (Sars M., 1870) & & 1 & & & + & + & + & + & & + & $\mathrm{SP}$ \\
\hline Solecardia rotunda & (Jeffreys, 1881) & & 2 & & & + & + & & & & & $\mathrm{SP}$ \\
\hline Montacuta substriata & (Montagu, 1808) & & 7 & + & & + & + & + & + & & & $\mathrm{SP}$ \\
\hline Kurtiella pellucida & (Jeffreys, 1881) & & 2 & + & & + & + & & & & & SP \\
\hline Kurtiella bidentata & (Montagu, 1803) & & 3 & + & & + & + & + & + & & & $\mathrm{SP}$ \\
\hline Epilepton clarkiae & (Clark W., 1852) & & 1 & + & & + & + & + & & & & SP \\
\hline Tellimya tenella & (Lovén, 1846) & 1 & 2 & + & & + & + & + & + & & & SP \\
\hline Parvicardium minimum & (Philippi, 1836) & 23 & 38 & + & FI & + & + & + & + & + & & SP \\
\hline Arcopagia balaustina & (Linnaeus, 1758) & 1 & 8 & + & $\mathrm{DE}$ & + & + & + & + & + & + & $\mathrm{SP}$ \\
\hline Tellina distorta & Poli, 1791 & 1 & & + & $\mathrm{DE}$ & + & + & + & + & + & + & $\mathrm{SP}$ \\
\hline Abra longicallus & (Scacchi, 1835) & 9 & 17 & + & $\mathrm{DE}$ & + & + & + & + & & & $\mathrm{SP}$ \\
\hline Kelliella miliaris & (Philippi, 1844) & 148 & 84 & + & FI & + & + & + & + & & & $\mathrm{SP}$ \\
\hline Pitar mediterranea & (Tiberi, 1855) & 1 & 1 & + & FI & + & + & + & & & + & SP \\
\hline Hiatella arctica & (Linnaeus, 1767) & & 3 & + & & + & + & + & + & + & & SP \\
\hline Saxicavella jeffreysi & Winckworth, 1930 & & 1 & & & + & + & + & + & + & & SP \\
\hline Thracia sp. & & 5 & 1 & & FI & & & & & & & $\mathrm{SP}$ \\
\hline Xylophaga dorsalis & (Turton, 1819) & & 1 & + & & + & + & + & + & + & & SP \\
\hline Panacca sp. & Dall, 1905 & 2 & 1 & & & & & & & & & $\mathrm{SP}$ \\
\hline Lyonsia norwegica & (Gmelin, 1791) & 1 & & & FI & + & + & + & + & + & & SP \\
\hline Verticordia granulata & Seguenza, 1860 & 6 & 15 & + & $\mathrm{CA}$ & + & + & + & + & + & & $\mathrm{SP}$ \\
\hline Polycordia gemma & (Verrill, 1880) & 1 & 1 & & & & & & & & & $\mathrm{SP}$ \\
\hline Poromya granulata & (Nyst and Westendorp, 1839) & 6 & 7 & & CA & + & + & + & + & + & + & SP \\
\hline Cuspidaria cuspidata & (Olivi, 1792) & 5 & 2 & + & $\mathrm{CA}$ & + & + & + & + & + & + & $\mathrm{SP}$ \\
\hline Cuspidaria rostrata & (Spengler, 1793) & 6 & 3 & + & $\mathrm{CA}$ & + & + & + & + & + & + & $\mathrm{SP}$ \\
\hline Tropidomya abbreviata & (Forbes, 1843) & 1 & 6 & + & $\mathrm{CA}$ & + & + & + & + & + & + & $\mathrm{SP}$ \\
\hline Cardiomya costellata & (Deshayes, 1833) & 66 & 30 & + & CA & + & + & + & + & + & + & SP \\
\hline SCAPHOPODA & & & & & & & & & & & & \\
\hline Antalis panorma & (Chenu, 1843) & 33 & 4 & & $\mathrm{CA}$ & + & + & + & & & + & SP \\
\hline Cadulus jeffreysi & (Monterosato, 1875) & 2 & 17 & & $\mathrm{CA}$ & + & + & + & + & & & $\mathrm{SP}$ \\
\hline Pulsellum lofotense & (M. Sars, 1865) & & 3 & & & + & + & + & + & & & $\mathrm{SP}$ \\
\hline
\end{tabular}




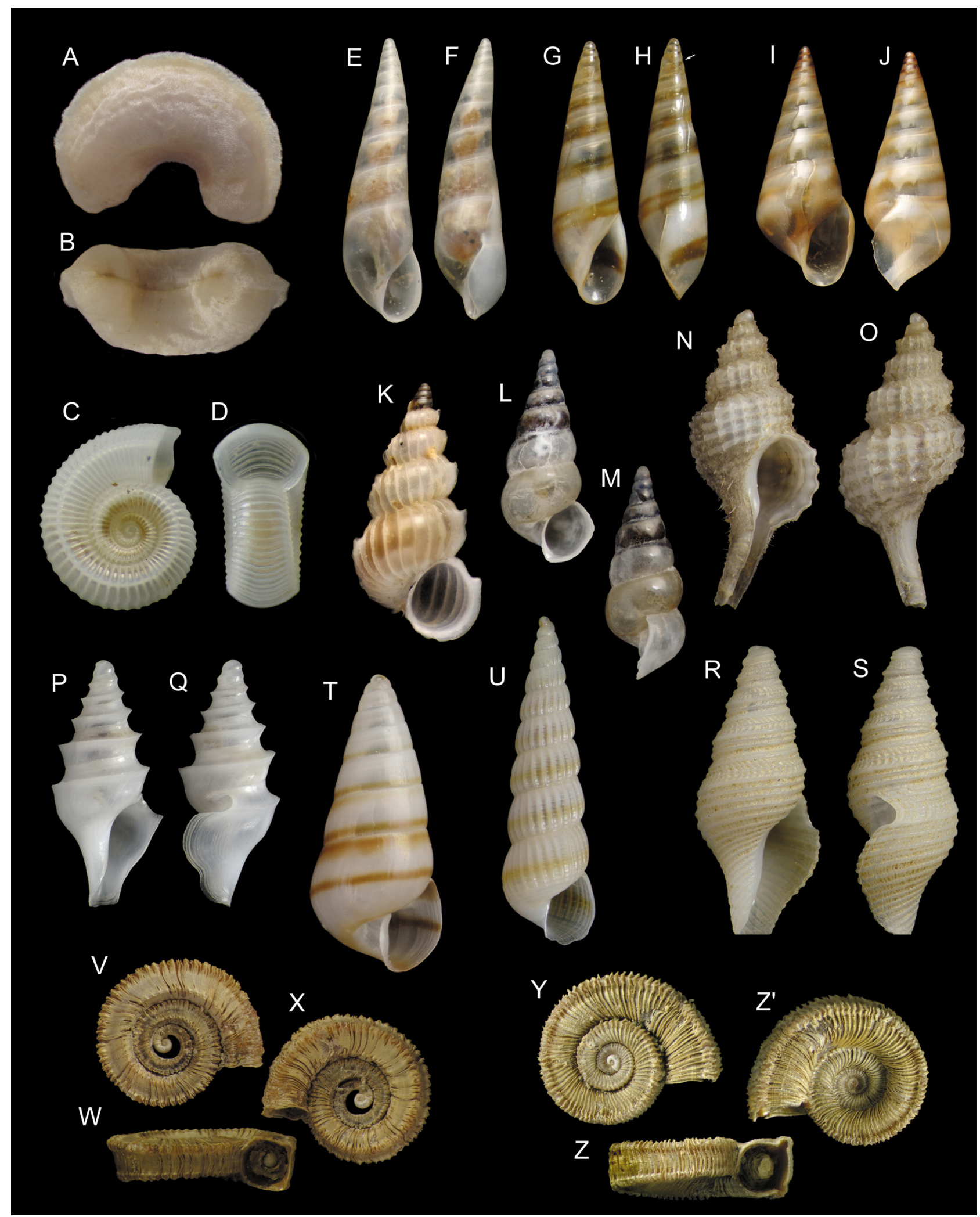

Fig. 4. - Some of the rare molluscs collected on Algarrobo Bank. A-B: Neomenia carinata (length $5.0 \mathrm{~mm}$ ). C-D: Adeuomphalus ammoniformis (maximum diameter $1.6 \mathrm{~mm}$ ). E-F: Curveulima devians (height $4.2 \mathrm{~mm}$ ). G-H: Eulima bilineata (height $4.0 \mathrm{~mm}$ ). I-J: Eulima fuscozonata (height $4.3 \mathrm{~mm}$ ). K: Epitonium hispidulum (height $3.4 \mathrm{~mm}$ ). L-M: Aclis attenuans (height $2.4 \mathrm{~mm}$ ). N-O: Trophonopsis barviciensis (height $7.3 \mathrm{~mm}$ ). P-Q: Spirotropis monterosatoi (height $9.2 \mathrm{~mm}$ ). R-S: Drilliola emendata (height $6.2 \mathrm{~mm}$ ). T: Tiberia minuscula (height $6.3 \mathrm{~mm}$ ); U: Turbonilla cf. vaillanti (height $7.4 \mathrm{~mm}$ ). V-X: Spirolaxis clenchi (maximum diameter $4.5 \mathrm{~mm}$ ). Y-Z': S. lamellifer (maximum diameter $5.3 \mathrm{~mm}$ ). 


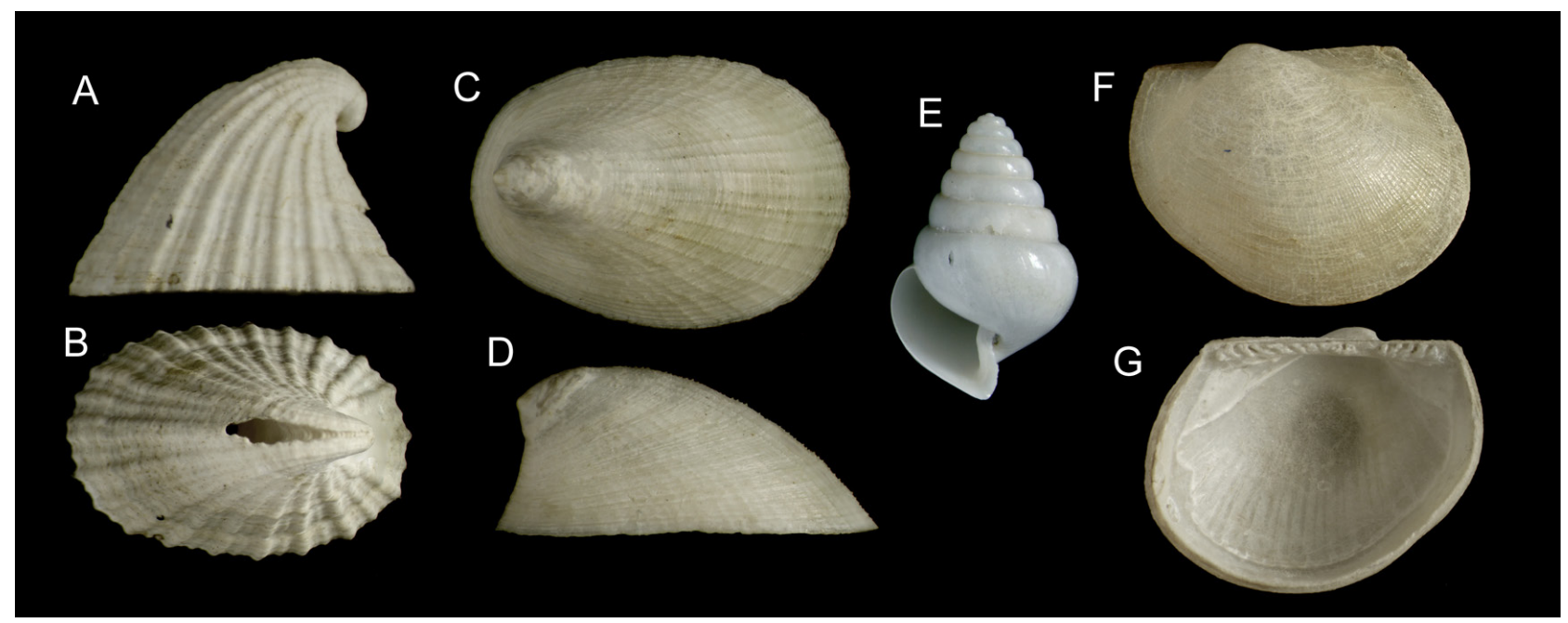

Fig. 5. - Molluscan species found only in thanatocoenosis on Algarrobo Bank. A-B: Puncturella noachina (length 5 mm). C-D: Iothia fulva (length $5.5 \mathrm{~mm}$ ). E: Limacina retroversa (height $2.8 \mathrm{~mm}$ ). F-G: Bathyarca frielei (length $8.5 \mathrm{~mm}$, compare with B. pectunculoides Fig. 3 F-G).

of the Shannon-Wiener diversity index $\mathrm{H}_{(\log 2)}^{\prime}=3.60$ and Pielou's evenness index J'=0.56.

Among molluscs, the dominant species of live specimens are Limopsis aurita $(676 ; \mathrm{D}=38.1 \%)$, Yoldiella philippiana $(275 ; \mathrm{D}=15.5 \%)$, Kelliella miliaris $(148 ; \mathrm{D}=8.3 \%)$, Bathyarca pectunculoides $(132$; $\mathrm{D}=7.4 \%)$, Pagodula echinata $(70 ; \mathrm{D}=3.9 \%)$ and Cardiomya costellata $(66 ; \mathrm{D}=3.7 \%)$, these species making up altogether $77 \%$ of the live molluscan specimens of the sediment fraction of the haul BT04 (Fig. 3). Gastropods slightly predominate in number of species (43 live-taken, 84 species in total) over bivalves (36 live-taken, 60 species in total) but in abundance the bivalves prevailed, with $73 \%$ of live-taken individuals, because of the overwhelming numbers contributed by Limopsis aurita.

There were 30 species represented by a single live specimen and 12 more represented by only two specimens. Some of the species found are considered rare or very rare (Fig. 4): the Solenogastres Neomenia carinata and Unciherpia hirsuta, the gastropods Adeuomphalus ammoniformis, Epitonium linctum, E. hispidulum, Eulima fuscozonata, Drilliola emendata, Spirolaxis clenchi, S. lamellifer, Tiberia minuscula and Philine monterosati, and the bivalves Crenella pellucida and Polycordia gemma. Two species are believed to be new to science and are described hereafter.

The gastropods Puncturella noachina, Anatoma tenuisculpta, Iothia fulva and Limacina retroversa, and the bivalve Bathyarca frielei were represented only by rather abundant shells, with a dull, opaque appearance and no live specimens of these species were collected (Fig. 5).

\section{Description of new taxa and remarks on some other rare species}

\section{Family EULIMIDAE \\ Genus Melanella}

(type species, Melanella dufresnii Bowdich, 1822, by monotypy)
Melanella scarifata Gofas and Rueda, n. sp. http://zoobank.org/F96DF946-94BB-4796-A143-D68070D7BAD0

Holotype: live-collected specimen from DEEPER 0409 cruise, BT04 (MNCN 15.05/60139).

Type locality: Algarrobo Bank, Alboran Sea $\left(36^{\circ} 21.08^{\prime} \mathrm{N}\right.$, $03^{\circ} 58.58^{\prime} \mathrm{E}, 349 \mathrm{~m}$ to $36^{\circ} 21.06^{\prime} \mathrm{N}, 03^{\circ} 58.07^{\prime} \mathrm{E}, 365 \mathrm{~m}$ )

Description. Shell small (holotype $5.1 \times 1.9 \mathrm{~mm}$ ) with a tall, very regularly conical spire, comprising in total 11 whorls, quite transparent in the early whorls but becoming gradually more opaque on the two last whorls. Height of last whorl (measured along the axis on Fig. 6A) $2.3 \mathrm{~mm}$ (45\% of total height), height of aperture $1.6 \mathrm{~mm}$, spire angle $25.5^{\circ}$.

Larval shell distinct, the visible part $530 \mu \mathrm{m}$ high and $350 \mu \mathrm{m}$ broad, transparent and thin, smooth, comprising 3.5 whorls which are slightly convex with a distinct suture. Teleoconch comprising 7.5 whorls, the spire whorls almost flat, the last whorl having its maximum convexity near the periphery and very slightly convex in the abapical part. Suture of teleoconch whorls not very conspicuous but distinct; parallel to this just beneath the upper $1 / 4$ of the whorls, there is a conspicuous internal suture (the "false suture") seen by transparency.

Axis very slightly bent, resulting in that the profile opposite the aperture (right side of Fig. 6B) is imperceptibly convex. Surface of teleoconch whorls apparently smooth, with a few hardly distinct growth stages, with very finely incised, opisthocline lines visible only under strong magnification, regularly spaced about $50 \mu \mathrm{m}$.

Aperture oblong, rounded in its abapical part; the columella and the parietal edge are bordered by a callus and form a very obtuse angle. Outer lip simple, slightly flaring so, in apertural view (Fig. 6A) the edge would reach slightly outside the prolongation of the spire profile. In lateral view, the outer lip of the aperture forms a small sinus just below the suture, then forms a regular curve with the most protruding part situated at the middle of its height. 


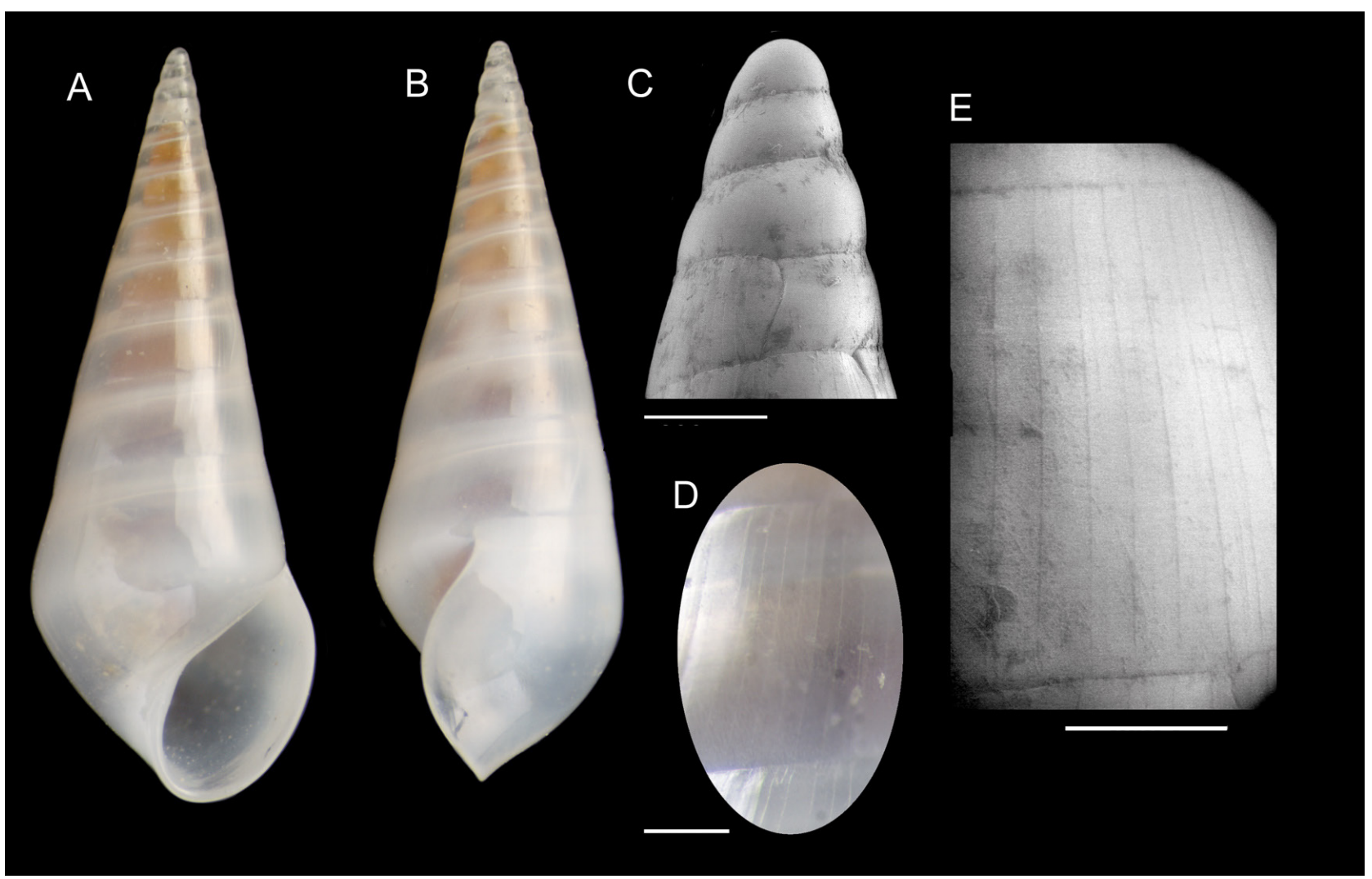

Fig. 6. - Holotype of Melanella scarifata Gofas and Rueda, n. sp. (height $5.1 \mathrm{~mm}$ ). A: apertural view. B: view showing the profile of the outer lip. C: protoconch, ESEM micrograph; note the flexuous scar demarcating protoconch from teleoconch. D: detail of the microsculpture under the optical stereomicroscope. E: ESEM micrograph of the microsculpture (scale bars are $200 \mu \mathrm{m}$ ).

Remarks. Eulimids commonly have rather featureless, smooth shells which renders them difficult to characterize at the species level. This species is unusual in having a definite microsculpture consisting of regularly spaced scars parallel to the growth lines. A similar sculpture is found in Melanella costellata (Dautzenberg and Fischer, 1897) from the bathyal of the Azores, and M. turbonilloides Bouchet and Warén, 1986 from abyssal depths of the North Atlantic; however, in these species the incisions are more pronounced, the shape of the spire is much more slender, with convex whorls on the teleoconch also, and the larval shell is paucispiral. Other species of European Melanella with a shell microsculpture include Melanella alba (da Costa, 1778), a much larger species (up to $20 \mathrm{~mm}$ ) and Melanella lubrica (Monterosato, 1890), only slightly larger (9 $\mathrm{mm}$ ) than the present species. Both of these species live in the infralittoral zone and are holothurian parasites (Warén 1984: 55, Gofas et al. 2011: 227-228). In the former, the microsculpture is also parallel to the growth lines but consists of densely crowded, minute wrinkles; in the latter the microsculpture is spiral (see Gofas et al. 2011: 227-228). The larval shell of Melanella scarifata $\mathrm{n}$. sp. is unusually large and denotes a planktotrophic larval development, so the species is probably not endemic to the Algarrobo Bank and may be found again in relatively remote places of the Atlantic and Mediterranean Sea.

Etymology. From Latin scarifare, to scratch, alluding to the microsculpture.
Family TOFANELLIDAE

Genus Graphis Jeffreys, 1867

(type species: Turbo unicus Montagu, 1803, by monotypy)

Graphis pruinosa Gofas and Rueda, n. sp. http://zoobank.org/8E244CB5-7AE6-4EA2-81D9-28CF10E80225

Holotype: live-collected specimen from DEEPER 0409 cruise, BT04 (MNCN 15.05/60140H); 7 paratypes (1 live-taken specimen and 6 shells) from the type locality (MNCN 15.05/60140P).

Type locality: Algarrobo Bank, Alboran Sea $\left(36^{\circ} 21.08^{\prime} \mathrm{N}\right.$, $03^{\circ} 58.58^{\prime} \mathrm{E}, 349 \mathrm{~m}$ to $36^{\circ} 21.06^{\prime} \mathrm{N}, 03^{\circ} 58.07^{\prime} \mathrm{E}, 365 \mathrm{~m}$ )

Description. Shell very small (holotype $2.1 \times 0.65$ $\mathrm{mm}$ ) with a tall spire, comprising in total six whorls, somewhat translucent but having a surface with a frosty appearance, not transparent. Height of last whorl (measured along the axis on Fig. 7A) $0.8 \mathrm{~mm}$ (38\% of total height), height of aperture $0.45 \mathrm{~mm}$, spire angle $11^{\circ}$. Colour whitish.

Protoconch $450 \mu \mathrm{m}$ high and $360 \mu \mathrm{m}$ broad, smooth, comprising hardly 1.5 whorls, the first of which is globose and bears a couple of blunt keels, one of which persists on the upper third the following half-whorl.

Teleoconch comprising 4.5 convex whorls, increasing very slowly in diameter, with a deeply impressed suture. Sculpture of teleoconch whorls composed of weak, irregular wrinkles parallel to the growth lines, flexuose, and of a few growth scars; there are very faint and shallow spiral microstriae, spaced ca. $5 \mu \mathrm{m}$ and detectable only under very high magnification. Aperture 


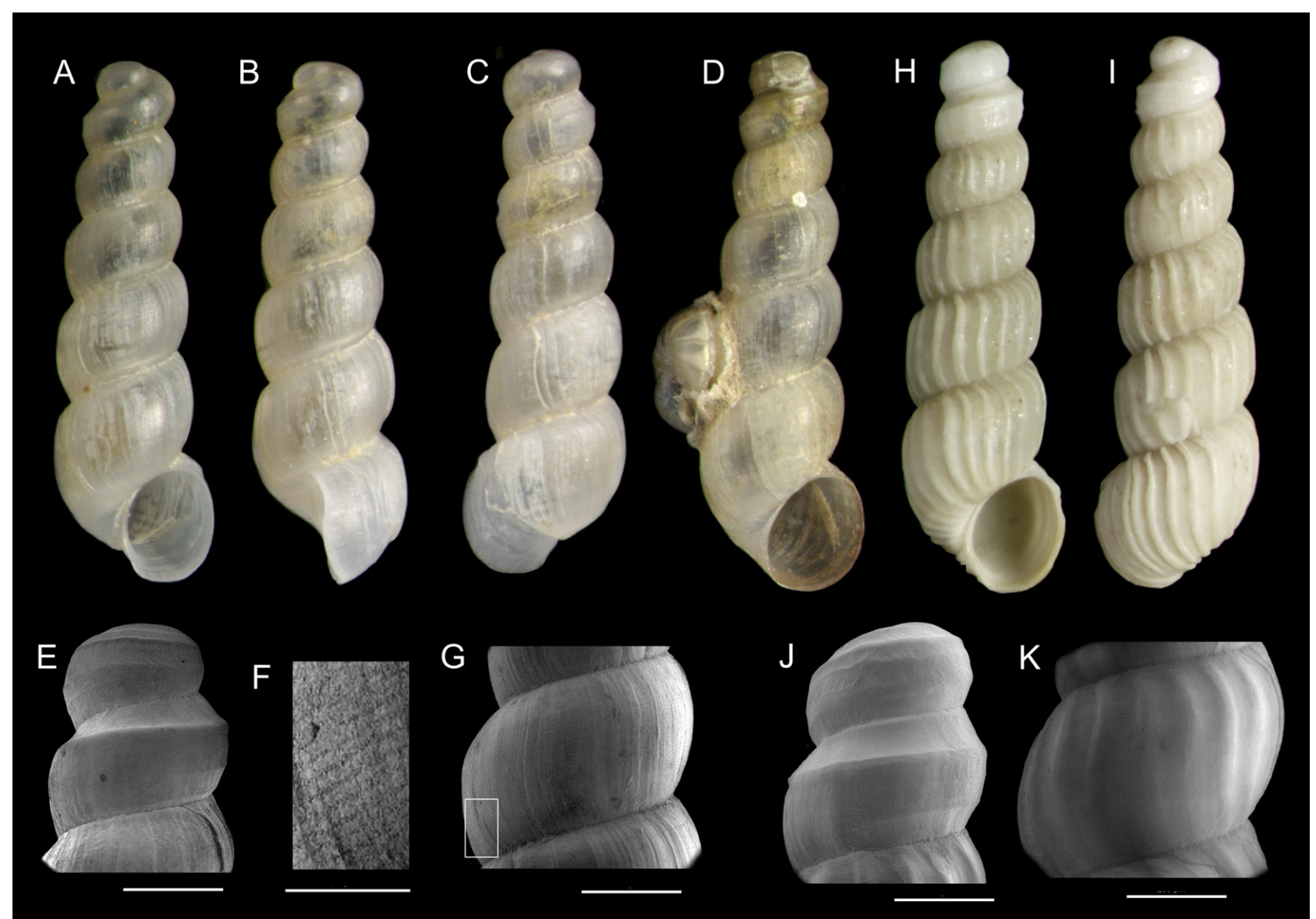

Fig. 7. - Graphis spp. from haul BT04. A-C: Holotype of Graphis pruinosa Gofas and Rueda, n. sp. (height $2.1 \mathrm{~mm}$ ). D: paratype, live collected (height $2.2 \mathrm{~mm}$ ); note the foraminifer Cibicidoides sp. attached. E. ESEM micrograph of the protoconch of another paratype (shell). F-G: ESEM micrographs, detail of the penultimate whorl of the holotype; the highly magnified area in F corresponds to the rectangle on G. H-I: Shell of Graphis gracilis Monterosato, 1874 (height $2.3 \mathrm{~mm}$ ). J: ESEM micrograph of the protoconch of another shell of Graphis gracilis. K: ESEM micrograph of the penultimate whorl, same shell as J. Scale bars for E, G, J, K: $200 \mu \mathrm{m}$, for F: $50 \mu \mathrm{m}$ ).

oval, with a continuous peristome, the outer lip simple, very slightly flaring, flexuous in lateral view (Fig. 7B); the columellar edge appressed.

Remarks. This species most resembles Graphis gracilis (Monterosato, 1874), from which it differs essentially by the absence of the strong axial ribs and the surface texture, which is shagreened rather than smooth, and under scanning electron microscope examination bears microstriae which are not present in the former. The protoconchs are essentially alike and this shared morphology indicates that these two species are probably related. Both species were found together on Algarrobo Bank, but G. gracilis was not collected alive.

Etymology. From Latin pruinosus, frosted, alluding to the aspect of the shell surface.

\section{Anatoma tenuisculpta (Seguenza, 1880)}

This species was reported, under the name Anatoma crispata (Fleming, 1828), from the Alboran platform as shells only by Peñas et al. (2006) but was then misidentified according to Geiger (2012). Numerous shells only were found in haul BT04, but living specimens were subsequently found on the Alboran platform (un- published data from the INDEMARES project), so the species is still extant in the area.

\section{Turbonilla cf. vaillanti}

Dautzenberg and Fischer, 1896

The presence of spiral sculpture and of a colour pattern on this species considerably limits the scope of possible comparisons among the nearly one hundred species of Turbonilla currently reported from European and western African coasts. Turbonilla vaillanti, originally described from the bathyal of the Azores and also reported from the Irving seamount (Peñas and Rolán 1999), is possibly the most similar species, with size and sculpture matching those of the present species. There are several differences: T. vaillanti is white, and the ribs are somewhat more prosocline on the early whorls. Nevertheless, we have observed several cases (e.g. the rissoid Alvania testae [Aradas and Maggiore, 1844], the columbellid Amphissa acutecostata [Philippi, 1844]) where the same species are white in deep-water and have a colour pattern in shallower environments. Therefore, although we cannot preclude that this is an undescribed species; we prefer to refer it as Turbonilla cf. vaillanti, in any case a new item for the Mediterranean fauna. 


\section{DISCUSSION}

\section{Numbers of species, diversity and new records}

The Mediterranean deep sea is infamous for the poverty of its fauna (Fredj and Laubier 1985, Bouchet and Taviani 1992, Galil 2004), to the point that it once provided support towards Forbes' (1844) "azoic theory". However, ongoing exploration reveals that some areas may be not so poor (Koutsoubas et al. 2000; Sardá et al. 2004; Danovaro et al. 2010). The number of species reported here (156 spp.) is nevertheless unusual for the Mediterranean deep sea, usually reported as being less diverse than other adjacent basins such as the Atlantic Ocean. At similar depths, thanatocoenoses were reported as "rich" with 61 species off NW Sicily (Di Geronimo et al. 2005). In the area of S. Maria di Leuca, off SE Italy, Negri and Corselli (2011) reported as many as 112 species of molluscs, but this was in the context of a broader sampling including 24 different box cores, not at a single site as here.

The BT04 haul is outstanding at the local scale but this may be an artefact of a particularly successful operation. Of the other two beam trawl operations on Algarrobo Bank, one (BT03) was void and one (BT05) got stuck on the bottom shortly after landing although it managed to collect a small amount of Leptometra. The other four beam trawls on the nearby Ville de Djibouti Bank yielded a considerably lower number of species and did not bring up a noteworthy amount of sediment to be sorted. The other gears are less effective at collecting benthic molluscs: the otter trawl operations are essentially targeted to fish and usually bring up a limited sample of small invertebrates, and the rock dredge collects a considerably smaller sample and covers a much smaller area $\left(150 \mathrm{~m}^{2}\right.$ versus $\left.800 \mathrm{~m}^{2}\right)$ of the bottom.

The solenogastre Unciherpia hirsuta (Pedrouzo et al. 2014) and the gastropods Spirotropis monterosatoi and Turbonilla cf. vaillanti are the first findings of these species in the Mediterranean fauna. The specimen of Adeuomphalus ammoniformis is the first unquestionable record of this species living in the Mediterranean, whereas previous records are suspected of being based on Pleistocene subfossil specimens (Kano et al. 2009). Likewise, the occurrence of several live-taken Trophonopsis barviciensis proves the presence of this species in the Recent Mediterranean fauna, which had been questioned by Bouchet and Warén (1985). Spirolaxis lamellifer and $S$. clenchi are the first specimens collected in Iberian waters.

The proportion of species of molluses which would have been overlooked if the processing were limited to the shipboard sorting is considerable, since only seven species were recovered at that stage (Table 1). Admittedly, the dominant (Limopsis aurita) and characteristic (Antalis panorma) species were correctly represented, but over $90 \%$ of the species were found only after sorting the fine-fraction residue. Conversely, two of the megafaunal species (Heliacus fallaciosus and Xenophora crispa) were not found again in the residue. This bias is particularly acute for molluscs, which are

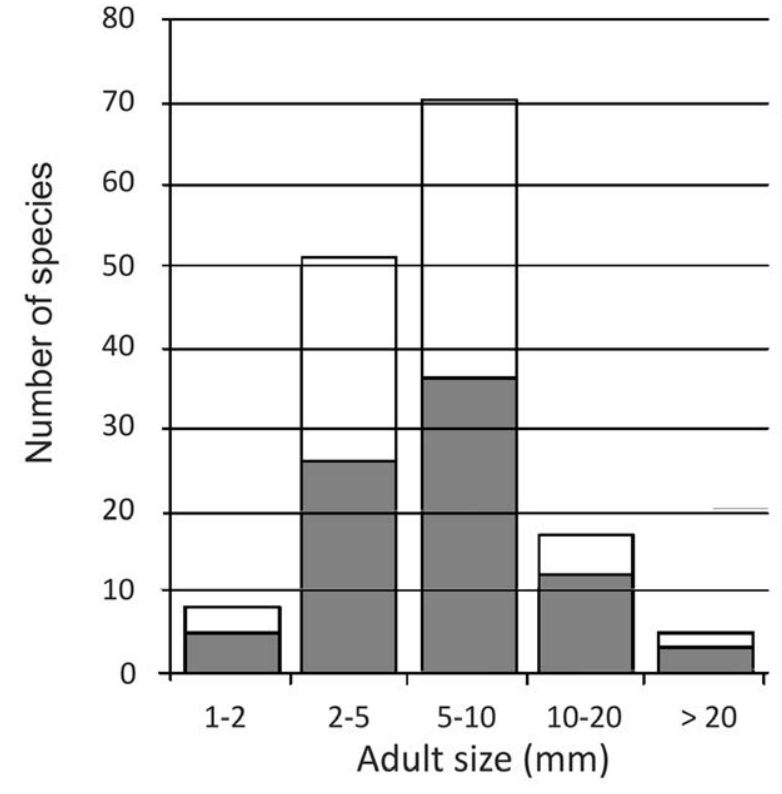

Fig. 8. - Histogram of size classes for the molluscan species represented in haul BT04: (bars represent the total number, shaded portion represents live collected species). Only the largest size classes (above $10 \mathrm{~mm}$ ) are expected to be represented in the shipboard sorting.

mostly represented by small species (Fig. 8). Streftaris and Zenetos (2007) reached a similar conclusion after analysing the molluscan species composition in a suite of samples from the NE Aegean Sea separately in the size fractions $>1 \mathrm{~mm}$ and $0.5-1 \mathrm{~mm}$ : among a total of 124 species, $84(67.7 \%)$ were encountered in the $>1 \mathrm{~mm}$ fraction, and $107(86.3 \%)$ in the $0.5-1 \mathrm{~mm}$ fraction. Groups such as fish and echinoderms are not in this case, but the bias may also be important for crustaceans, where the small peracarids would contribute many species in addition to the megafaunal decapods. This observation highlights the importance of laboratory sorting in order to obtain a complete assessment of species richness and diversity, most of which may be missed if the processing is limited to shipboard observations.

It is striking that one-third (54 out of 156) of the species found on Algarrobo Bank were not reported in the large species list (655 spp.) from the surroundings of Alboran Island (Peñas et al. 2006). The number of nonshared species was slightly reduced by the recent and intensive exploration of Alboran in the INDEMARES project, which added Neomenia carinata and Pitar mediterranea to the list of shared species, in a dataset of 191 live-collected mollusc species (4.331 specimens). The Alboran platform holds much more diverse habitats, with a much broader bathymetric range which starts from the supralittoral (Templado et al. 2006) and therefore it is straightforward that many of the species found in the shallower parts of this platform should not be found on Algarrobo Bank. Conversely the presence on the bank of a high number of species not shared with the Alboran platform may be related to a deeper summit (ca. $350 \mathrm{~m}$ ) than the Alboran platform (ca. 50-200 $\mathrm{m})$, but it can be also indicate genuine heterogeneity among different sectors of the Alboran Sea. 


\section{Biogeographic affinity of the molluscan fauna}

The bulk of the species found on Djibouti Banks have a more or less extensive range in the Atlantic and most of them were also previously known in the
Mediterranean. An analysis of the molluscan fauna using presence/absence in the six regions listed in Table 2 and the Bray-Curtis similarity index (Fig. 9) shows that 103 of the species cluster together with a similarity above $75 \%$, with various combinations of ranges

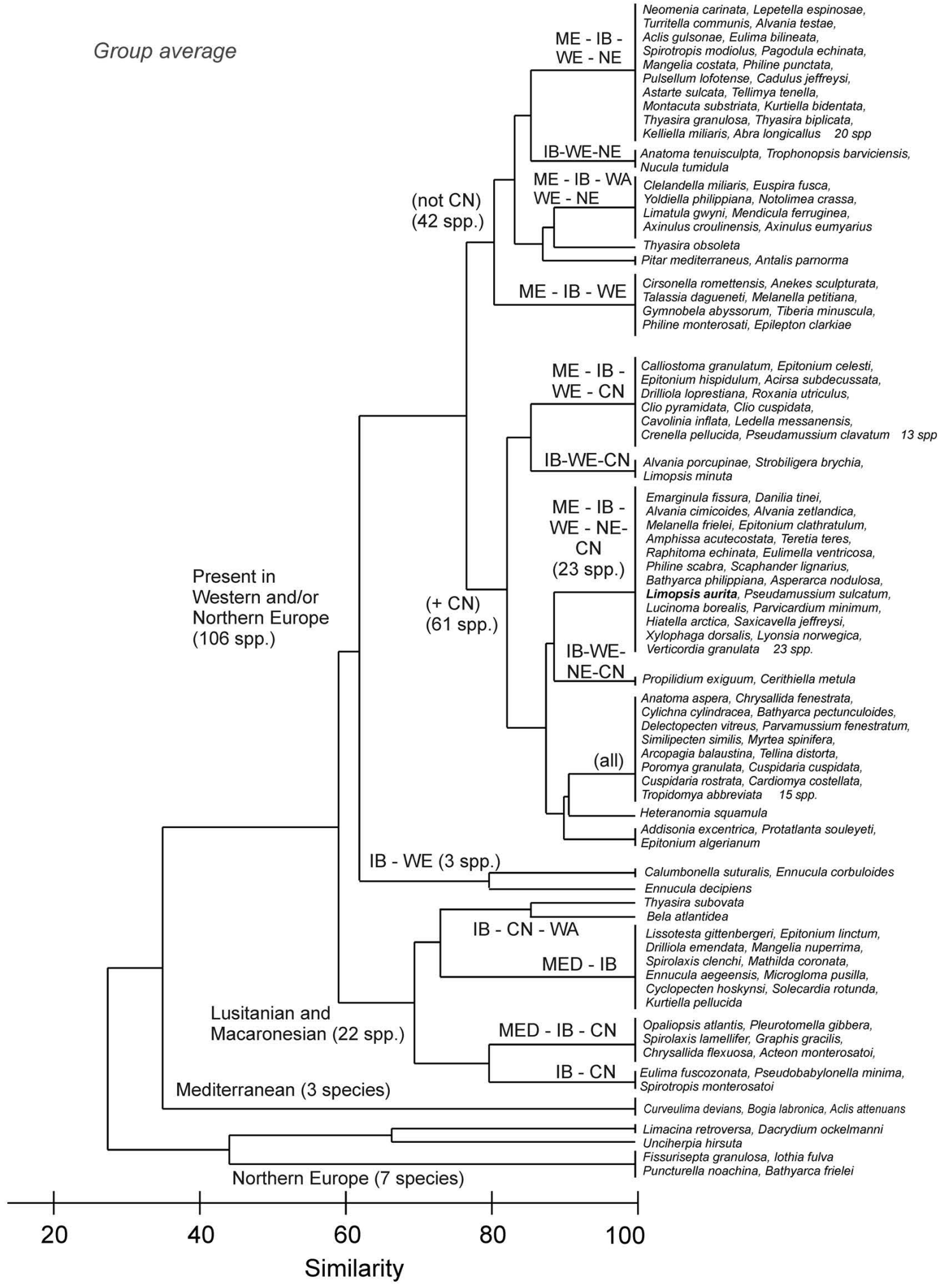

Fig. 9. - Cluster of faunal groups from different geographic areas represented in haul BT04, obtained using presence/absence data and the Bray-Curtis similarity index. (ME: Mediterranean; IB: Ibero-Moroccan Gulf; WE: Western Europe; NE: Northern Europe; CN: Canary Islands, Madeira and Lusitanian seamounts, WA: West Africa). 

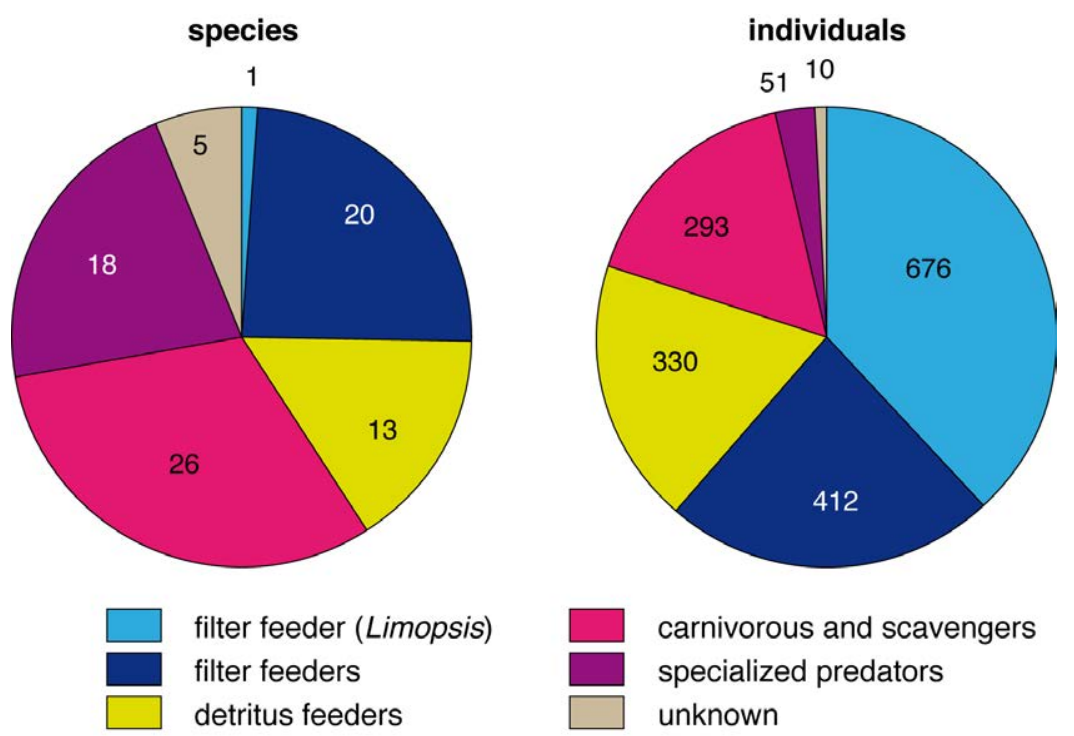

Fig. 10. - Distribution of molluscan species and of individuals by feeding guilds, in haul BT04.

involving western and/or NE. On the other hand, only three species (Curveulima devians, Aclis attenuans and Bogia labronica) are reported in the Mediterranean outside the Alboran Sea but not in the Atlantic. A similar biogeographical pattern of coastal molluscan assemblages was found by Rueda et al (2009) and Marina et al. (2012), indicating the importance of species with a broad Atlantic range in the bulk of fauna at different depth levels in the Alboran Sea.

A small group of species (Puncturella noachina, Iothia fulva, Limacina retroversa, Bathyarca frielei, Dacrydium ockelmanni) cluster together with a distribution currently restricted to northern and western Europe and not reported in the Ibero-Moroccan area. The three former are cited by Malatesta and Zarlenga (1986) as Mediterranean "cold hosts" during cold climatic epochs and are here interpreted as elements of a thanatocoenosis, now extinct in the Mediterranean Sea. Indeed, abundant cold-water coral remains occur at the summit of this and other banks, and related to high productivity during the Late Pleistocene and Holocene (Hebbeln et al. 2009, Fink et al. 2013).

Regarding potential for dispersal, the bulk of the species found in haul BT04 have a planktonic stage, albeit not always planktotrophic. Of the molluscan species taken alive, only Trophonopsis barvicensis, Pagodula echinata, Pseudobabylonella minima, Drilliola emendata, Spirotropis monterosatoi and Graphis pruinosa n. sp. are inferred to have direct development. Therefore, it can be hypothesized that the fauna benefits from a diverse influx of larvae carried in by the incoming Atlantic current. The Atlantic water is not directly in contact with the bottom but sufficiently close for sinking larvae to reach the bank and settle there. Obviously, the higher salinity of the Levantine Intermediate Water in contact with the summit of Algarrobo Bank, which Bouchet and Taviani (1992) put forward as the second "barrier" after the sill of Gibraltar, is not so severe as it could be overcome by a large number of species. GarcíaMuñoz et al. (2012) indicated the presence of low hydrodynamic conditions and a high incidence of pelagic sedimentation at the summit of Algarrobo and Herradura banks based on sedimentological characteristics and this may benefit the vertical influx of larvae. Moreover, the dense population of Leptometra phalangium at the summit, as suspension feeders, benefit from a highly productive environment due to a high amount of particles from surficial levels (Colloca et al. 2004, Gil et al. 2010).

\section{Trophic groups and characterization of the taxocenosis}

Regarding the species level, there is a considerable balance between the different trophic groups, with a large representation of specialized carnivores or ectoparasites such as the families Eulimidae or Epitoniidae. When the counts are made at the individual level, the balance is biased towards suspension feeders, because of the dominance of the single species Limopsis aurita (Fig. 10). Taking into account that the structuring species Leptometra phalangium is itself a suspension feeder, the abundance of this group suggest that there must be a good supply of particles at the summit of the bank. The other abundant trophic group is the detritus feeders, which together with the filter feeding form the base of the animal food web in the Algarrobo Bank, with its summit in the aphotic zone and therefore without the presence of primary producers. The latter, however, indirectly influence the food web in the bank because the detritus down from the photic zone constitutes the main source of energy in the bank. According to Moore et al (2004), the detritus-based food web results in a more diverse, stable and structured community, particularly for ectotherm organisms, because the detritus represents energy persistent in time.

The specialized predators are represented by many rare species, so they account for $18 \%$ of the species but only $2.5 \%$ of the individuals, suggesting a high faunal diversity but with low population density. On the other hand, a broad representation of trophic groups 
is considered as an indicator of a stable environment (Sanders 1968, 1979, Levinton 1972)

\section{Habitat characterization}

The structuring species Leptometra phalangium and the scaphopod Antalis panorma are reported as exclusive characteristic species of the offshore detritic bottom community ("Détritique du Large", Pérès and Picard 1954, 1964: 94, Bellan-Santini et al. 2002). The bivalves Pseudamussium clavatum and Astarte sulcata, also reported as characteristic, were represented only by shells in our material. Unlike "Detritique Côtier", where some facies may incorporate several species of algae including rhodolith-forming algae, benthic photosynthetic organisms are never involved in the community of the "Détritique du Large". It is disputed whether this community should be encompassed in the circalittoral or in the bathyal stage. Formally speaking, it belongs to the aphytal zone, and therefore to the bathyal (the view defended by Emig 1997). Nevertheless, Pérès and Picard (1964) and Pérès (1982: 479) advocated grouping it with the circalittoral because so many animal species are shared with that stage. Our molluscan species list definitely supports the former view: it contains hardly any species that would normally occur shallower than $200 \mathrm{~m}$ (Arcopagia balaustina and Tellina distorta would be the only ones in this case and these are only represented by one live-collected specimen).

\section{Conservation aspects}

Because of its exceptional richness, both in numbers and in the quality of the species, this type of bathyal community with Leptometra and Limopsis should be considered a high priority for habitat conservation in the Mediterranean deep sea. Mediterranean communities of the offshore "detritic" bottoms have also attracted interest elsewhere and qualify as a "reservoir" of biodiversity (Colloca et al. 2004, 2006).

Circalittoral, shelf-edge and upper slope gravel bottoms are a primary target for trawling activities because (1) they are mostly situated below the legal limit of $50 \mathrm{~m}$ for this activity, (2) the sediment forms extensive stretches amenable to trawling and (3) many species of fishes and cephalopods, which predominate in trawling shelf landings, seem to be related to these beds. Trawling can be intuitively perceived as a threat to the benthic assemblages, particularly to the epifauna, and where quantitative data exist it has effectively been recognized as a pressure affecting this community (Mangano et al. 2013).

Particular attention is needed for the shelf break facies with abundant Leptometra phalangium, which is increasingly perceived as the habitat of an exceptionally diverse benthic fauna that is easily disturbed by trawling. The outstanding species richness may be favoured because the crinoid increases heterogeneity, but above all its presence is an indicator of favourable resources and it has been explicitly put forward as a species deserving a protection status (Colloca et al. 2004, De Ranieri et al. 2006, Angeletti et al. 2010). A strong case has been made for the recognition of Leptometra phalangium aggregates as Essential Fish Habitats by the working group (SGMED 06-01) of the scientific, technical and economic committee for fisheries (European Commission 2006), with several papers on the subject presented at the 2006 meeting (Abelló and Gil de Sola 2006, Colloca et al. 2006). This culminated in having Leptometra beds (along with Posidonia beds 1120, a priority habitat in Europe's Habitat Directive, and along with maërl beds) as one of the 14 habitats selected by the Scientific Advisory Committee of the General Fisheries Commission for the Mediterranean as criteria for identifying Essential Marine Habitats $(\mathrm{EMH})$ of relevance for the management of priority species (General Fisheries Commission for the Mediterranean 2009). We conclude that it is unfortunate that the area investigated herein was not included in the final proposal for deep-water Sites of Community Interest in Spanish waters.

\section{ACKNOWLEDGEMENTS}

This is a contribution from the DEEPER project of the Instituto Español de Oceanografía. The access to unpublished data of the INDEMARES Alboran project (Life+ INDEMARES) is here acknowledged. The ESEM micrographs were taken at the Centro Andaluz de Nanomedicina y Biotecnología (BIONAND), Málaga, by Juan Félix López Téllez. We also gladly acknowledge the contribution of the crew of R/V Cornide de Saavedra to the success of the expedition to the Djibouti Banks.

\section{REFERENCES}

Abelló P., Gil de Sola L. 2006. Population characteristics of decapod crustaceans associated to Leptometra phalangium bottoms off the Iberian Peninsula Mediterranean coasts. In: Report of the Mediterranean subgroup (SGMED 06-01) of the Scientific, Technical and Economic Committee for Fisheries (STECF). Sensitive and Essential Fish Habitats in the Mediterranean Sea. Rome 6-10 March 2006, pp. 49-66.

Angeletti L., Ceregato A., Ghirelli M., et al. 2010. ROV-SCUBA integrated survey of the Montecristo Island Nature Reserve (Tuscan Archipelago National Park, Mediterranean Sea). Int. J. Soc. Underw.Technol. 29(3): 1-4.

Appeltans W., Ahyong S.T., Anderson G., et al. 2012. The magnitude of global marine species diversity. Curr. Biol. 22(23): 2189-2202. http://dx.doi.org/10.1016/j.cub.2012.09.036

Baro J., Rueda J.L., Díaz-del-Río V. 2012. South Iberian submarine canyons in the Alboran Sea: geohabitats, associated communities and fisheries resources. In: Wurtz M. (ed.), Mediterranean Submarine Canyons: Ecology and Governance. IUCN, Gland, Switzerland and Málaga, Spain, pp. 145-156.

Bedulli D., Bassignani F., Bruschi A. 2002. Use of biodiversity hotspots for conservation of marine molluscs: a regional approach. Med. Mar. Sci. 3(2): 113-121.

Bellan-Santini D., Bellan G., Bitar G., et al. 2002. Handbook for interpreting types of marine habitat for the selection of sites to be included in the national inventories of natural sites of conservation interest. Tunis: United Nations Environment Programme, Action Plan for the Mediterranean, Regional Activity Centre for Specially Protected Areas, $217 \mathrm{pp}$.

Bouchet P., Taviani M. 1992. The Mediterranean deep-sea fauna: pseudopopulations of Atlantic species? Deep-Sea Res. 39(2): 169-184. http://dx.doi.org/10.1016/0198-0149(92)90103-Z

Bouchet P., Warén A. 1980. Revision of the North-East Atlantic bathyal and abyssal Turridae (Mollusca: Gastropoda). J. Moll. Stud. Suppl. 8: 1-119. 
http://dx.doi.org/10.1093/mollus/46.Supplement_8.1

Bouchet P. Warén A 1985. Revision of the Northeast Atlantic bathyal and abyssal Neogastropoda excluding Turridae (Mollusca, Gastropoda). Boll. Malac. Suppl. 1: 121-296.

Bouchet P., Warén A. 1986. Revision of the Northeast Atlantic bathyal and abyssal Aclididae, Eulimidae, Epitonidae (Mollusca, Gastropoda). Boll. Malac. Suppl. 2: 297-576.

Bouchet P., Warén A. 1993. Revision of the Northeast Atlantic bathyal and abyssal Mesogastropoda. Boll. Malac. Suppl. 3: 579-840.

Coll M., Piroddi C., Steenbeek J., et al. 2010. The biodiversity of the Mediterranean Sea: estimates, patterns, and threats. PLoS One, 5(8): e11842. http://dx.doi.org/10.1371/journal.pone.0011842

Colloca F., Carpentieri P., Balestri E., et al. 2004. A critical habitat for Mediterranean fish resources: shelf-break areas with Leptometra phalangium (Echinodermata: Crinoidea). Mar. Biol. 145(6), 1129-1142. http://dx.doi.org/10.1007/s00227-004-1405-8

Colloca F., Sartor P., Baino R., et al. 2006. Crinoid beds as Essential Fish Habitat in the Tyrrhenian - Ligurian sea area (Fao Geographical sub-area 9). In: Report of the Mediterranean subgroup (SGMED 06-01) of the Scientific, Technical and Economic Committee for Fisheries (STECF). Sensitive and essential fish habitats in the Mediterranean Sea. Rome 6-10 March 2006, pp. 128-146,

Danovaro R., Company J.B., Corinaldesi C., et al. 2010. DeepSea Biodiversity in the Mediterranean Sea: The known, the unknown, and the unknowable. PLoS ONE 5(8): e11832. doi:10.1371/journal.pone.0011832 http://dx.doi.org/10.1371/journal.pone.0011832

De Ranieri S., Reale B., Ligas A., et al. 2006. Caratterizzazione della fauna associata alla facies a Leptometra phalangium (J. Muller, 1841) (Echinodermata; Crinoidea) nel mar Tirreno settentrionale. In: Domevici V., Lenzi A. (eds), Codice Armonico, Primo congresso di scienze naturali della Regione Toscana. Museo di Storia Naturale di Rosignano Solvay, Castiglioncello, Italy, pp. 32-36.

Di Geronimo I., Messina C., Rosso A., et al. 2005. Enhanced biodiversity in the deep: Early Pleistocene coral communities from southern Italy. In: Freiwald A., Roberts J.M. (eds), Cold-Water Corals and Ecosystems. Springer, Berlin Heidelberg, pp. 61-86. http://dx.doi.org/10.1007/3-540-27673-4 4

Emig C. 1997. Bathyal zones of the Mediterranean continental slope: An attempt. Publ. Esp., Inst. Esp. Oceanogr. 23: 23-33.

European Commission 2006 Report of the Mediterranean subgroup (SGMED 06-01) of the Scientific, Technical and Economic Committee for Fisheries (STECF). Sensitive and essential fish habitats in the Mediterranean Sea. Rome 6-10 March 2006, $48 \mathrm{pp}$, available online from http://stecf.jrc.ec.europa. eu/documents/43805/122924/06-04_SG-MED+06-01++Sensitive+habitats SECxxx.pdf

Fink H.G., Wienber C., de Pol-Holz R, et al. 2013. Cold-water coral growth in the Alboran Sea related to high productivity during the Late Pleistocene and Holocene. Mar. Geol. 339: 71-82. http://dx.doi.org/10.1016/j.margeo.2013.04.009

Forbes E. 1844. Report on the Mollusca and Radiata of the Aegean Sea, and on their distribution, considered as bearing on geology. Rep. Brit. Ass. Adv. Sci. 1843: 130-193.

Fredj G., Laubier L. 1985. The deep Mediterranean benthos. In: Moraitou Apostolopoulou M., Kuortsis V. (eds), Mediterranean marine ecosystems. NATO Conference series (I Ecology), Plenum Press, New-York, vol. 8, pp. 109-145.

Galil B.S. 2004. The limit of the sea: the bathyal fauna of the Levantine Sea. Sci. Mar. 68 (S3): 63-72. http://dx.doi.org/10.3989/scimar.2004.68s363

García-Muñoz M., López-González N., Rueda J.L., et al. 2012. Caracterización ambiental de los montes submarinos del Mar de Alborán a partir del estudio de los sedimentos y las asociaciones de foraminíferos bentónicos. Geogaceta 52: 165-168.

Geiger D.L. 2012. Monograph of the little slit shells. Volume 1. Introduction, Scissurellidae. pp. 1-728. Volume 2. Anatomidae, Larocheidae, Depressizonidae, Sutilizonidae, Temnocinclidae. pp. 729-1291. Santa Barbara Mus. Nat. Hist. Monogr. 7.

General Fisheries Commission for the Mediterranean. 2009. Meeting of the Sub-Committee on Marine Environment and Ecosystems (SCMEE), Malaga, Spain, 30 November - 3 December 2009. Criteria for the identification of sensitive habitats of relevance for the management of priority species. Available online <http://151.1.154.86/GfcmWebSite/SAC/SCMEE/2009/
Criteria sensitive habitat.pdf>

Gennari G., Spezzaferri S., Comas M.C., et al. 2013. Sedimentary sources of the mud-breccia and mud volcanic activity in the Western Alboran Basin. Mar. Geol. 339: 83-95. http://dx doi.org/10.1016/j.margeo.2013.04.002

Gil J., Bárcenas P., Baro J., et al. 2009. Caracterización preliminar de los sedimentos superficiales de los Bancos de Djibouti, Mar de Alborán (resultados de la campaña DEEPER0908). In: Troncoso J.S., Alejo I., López J. (eds), Resumenes II Simp. Int. Ciencias del Mar, 27-30 April 2009, Vigo, pp 260-261

Gil J., Rueda J.L., Baro J., et al. and the DEEPER team. 2010. Composición y estructura de la comunidad bentónico-demersal de montes submarinos del Mar de Alborán. In: Bayle Sempere J. (coord.) Resumenes XVI Simp. Ibérico Est. Biol. Mar. (España), 6-10 September 2010, Alicante, p. 126

Gladstone W. 2002. The potential value of indicator groups in the selection of marine reserves. Biol. Conserv. 104: 211-220. http://dx.doi.org/10.1016/S0006-3207(01)00167-7

Gofas S., Moreno D., Salas C. (coords.). 2011. Moluscos marinos de Andalucía. Volumen I, pp. i-xvi, 1-342; Volumen II, pp. i-xii, 343-798. Servicio de Publicaciones e Intercambio Científico, Universidad de Málaga.

Hebbeln D., Wienberg C., Beuck L., et al. and cruise participants. 2009. Report and preliminary results of RV POSEIDON Cruise POS 385 "Cold-Water Corals of the Alboran Sea (western Mediterranean Sea)", Faro - Toulon, May 29 - June 16, 2009. Berichte, Fachbereich Geowissenschaften, Universität Bremen, 273: 79 pp. Available online from: https://www.marum.de/Binaries/Binary5309/MARUM POS385 cruise report.pd

Hernández J.M., Rolán E., Swinnēn F., et al. 2011. Moluscos y conchas marinas de Canarias. ConchBooks, Hackenheim, 716 pp.

IEO-MAGRAMA 2012. Estrategia Marina. Demarcación Marina Estrecho y Alborán. Parte 4: Descriptores del buen estado ambiental. Descriptor 6: Fondos Marinos. Evaluación inicial y buen estado ambiental. Ministerio de Agricultura, Alimentación y Medio Ambiente, Secretaría General Técnica, Centro de Publicaciones, 41 pp. Available online from http://www. magrama.gob.es/es/costas/temas/estrategias-marinas/IV_D6_ Estrecho y Alboran tcm7-207244.pdf

IHO 2013. Standardization of undersea feature names: guidelines, proposal form, terminology Publication B-6, Edition 4.1.0, English/French Version. International Hydrographic Organization, Monaco, viii $+18+8 \mathrm{pp}$

Jablonski D., Lutz R.A. 1980. Molluscan larval shell morphology. Ecological and paleontological applications. In: Rhoads D.C., Lutz R.A. (eds), Skeletal growth of aquatic organisms. Plenum Press, New York, pp. 323-377. http://dx.doi.org/10.1007/978-1-4899-4995-0_10

Kano Y., Chikyu E., Warén A. 2009. Morphological, ecological and molecular characterization of the enigmatic planispiral snail genus Adeuomphalus (Vetigastropoda: Seguenzioidea). J. Moll. Stud. 75(4): 397-418. http://dx.doi.org/10.1093/mollus/eyp037

Koutsoubas D., Tselepides A., Eleftheriou A. 2000. Deep sea molluscan fauna of the Cretan Sea (Eastern Mediterranean): Faunal, ecological and zoogeographical remarks. Senckenb. Marit. 30 (3-6): 85-98. http://dx.doi.org/10.1007/BF03042958

Krebs C.J. 1989. Ecological methodology. Harper and Row Publishers, New York, $645 \mathrm{pp}$

Levinton J. 1972. Stability and trophic structure in deposit-feeding and suspension-feeding communities. Am. Nat. 106(950): 472-486. http://dx.doi.org/10.1086/282788

Lo Iacono C., Gràcia E., Diez S., et al. 2008. Seafloor characterization and backscatter variability of the Almería Margin (Alboran Sea, SW Mediterranean) based on high-resolution acoustic data. Mar. Geol. 250: 1-18. http://dx.doi.org/10.1016/j.margeo.2007.11.004

Malatesta A., Zarlenga F. 1986. Northern guests in the Pleistocene Mediterranean sea. Geol. Romana 25: 91-154.

Mangano M.C., Kaiser M.J., Porporato E.M.D., et al. 2013. Evidence of trawl disturbance on mega-epibenthic communities in the Southern Tyrrhenian Sea. Mar. Ecol. Prog. Ser. 475: 101-117. http://dx.doi.org/10.3354/meps 10115

Marina P. Urra J., Rueda J.L., et al. 2012. Composition and structure of the molluscan assemblage associated with a Cymodocea nodosa bed in south-eastern Spain: seasonal and diel variation. Helgol. Mar. Res. 66: 1-15. 
http://dx.doi.org/10.1007/s10152-012-0294-3

Millot C. 2009. Another description of the Mediterranean Sea outflow. Progr. Oceanogr. 82: 101-124. http://dx.doi.org/10.1016/j.pocean.2009.04.016

Millot C. 2013. Levantine Intermediate Water characteristics: an astounding general misunderstanding! Sci. Mar. 77(2): 217-232. http://dx.doi.org/10.3989/scimar.03518.13A

Moore J.C., Berlow, E.L., Coleman, D.C., et al. 2004. Detritus, trophic dynamics and biodiversity. Ecol. Lett. 7(7): 584-600. http://dx.doi.org/10.1111/j.1461-0248.2004.00606.x

Negri M.P., Corselli C. 2011. Mollusc associations from a coldwater coral environment (Apulian margin, S Italy). Bull. Geol. Soc. Finland, 83 (spec. iss. 1): 69

Palomino D., Vázquez J.T., Ercilla G., et al. 2011. Interaction between seabed morphology and water masses around the seamounts on the Motril Marginal Plateau (Alboran Sea, Western Mediterranean). Geo Mar. Lett. 31: 465-479 http://dx.doi.org/10.1007/s00367-011-0246-y

Pardo E., Aguilar R., García S., et al. 2011. Documentación de arrecifes de corales de agua fría en el Mediterráneo occidental (Mar de Alborán). Chronica Naturae 1: 20-34.

Parrilla G., Kinder T.H., Preller R.H. 1986. Deep and Intermediate Mediterranean Water in the western Alboran Sea. Deep-Sea Res. 33: 55-88. http://dx.doi.org/10.1016/0198-0149(86)90108-1

Pedrouzo L., Cobo M.C., García-Álvarez O., et al. 2014. Solenogastres (Mollusca) from expeditions off the South Iberian Peninsula, with the description of a new species. J. Nat. Hist. 48(45-48): 2985-3006. http://dx.doi.org/10.1080/00222933.2014.959576

Peñas A., Rolán E. 1999. Pyramidellidae (Gastropoda, Heterostropha) de la misión oceanográfica "Seamount 2". Iberus, suppl. 5: 151-199.

Peñas A., Rolán E., Luque A.A., et al. 2006. Moluscos marinos de la isla de Alborán. Iberus, 24(1): 23-151.

Pérès J.M. 1982. Major benthic assemblages. In: Kinne O. (ed.), Marine Ecology, Vol. V. Ocean management. J. Wiley \& Sons, London, Part 1, pp. 373-522.

Pérès J.M., Picard J. 1954. Les "fonds détritiques du large" à Leptometra phalangium et Dentalium panormum. C.R. Acad. Sci., Paris, 238: 1252-1254.

Pérès J.M., Picard J. 1964. Nouveau manuel de bionomie benthique de la Mer Mediterranée. Rec. Trav. Stat. Mar. Endoume 31(47): $1-138$.

Pielou E.C. 1969. An introduction to mathematical ecology. Wiley, New York, 286 pp.

Rueda J.L., Gofas S., Urra J., et al. 2009. A highly diverse molluscan assemblage associated with eelgrass beds (Zostera marina L.) in the Alboran Sea: Micro-habitat preference, feeding guilds and biogeographical distribution. Sci. Mar. 73(4): 679-700. http://dx.doi.org/10.3989/scimar.2009.73n4679

Salas C. 1996. Marine Bivalves from off the Southern Iberian Peninsula collected by the Balgim and Fauna 1 expeditions. Haliotis 25: 33-100.

Samadi S., Schlacher T., Richer de Forges B. 2007. Seamount benthos. In: Pitcher T.J., Morato T., Hart P.J.B., et al. (eds), Seamounts: Ecology, Fisheries, and Conservation. Blackwell, Oxford, 119-140.

Sanders H.L. 1968. Marine benthic diversity: a comparative study. Am. Nat. 102 (925): 243-282 http://dx.doi.org/10.1086/282541

Sanders H.L. 1979. Evolutionary ecology and life-history patterns in the deep sea. Sarsia 64(1-2): 1-7.

Sardà F., Calafat F., Flexas M., et al. 2004. An introduction to Mediterranean deep-sea biology. Sci. Mar. 68(Suppl. 3): 67-38.

Secretaría General de Pesca Marítima, 2004. Mar Mediterráneo. Costa Sur de España-Mar de Alborán (MA-6), de Longitud $-3^{\circ} 24^{\prime}$ a $-2^{\circ} 45^{\prime}$ y latitud $36^{\circ} 02^{\prime}$ a $35^{\circ} 41.5^{\prime}$, según los levantamientos efectuados con ecosonda multihaz por el B/O Vizconde de Eza (SGPM) en el año 2002 y 2003. Ministerio de Agricultura, Pesca y Alimentación, Madrid.

Smith S.D.A. 2005. Rapid assessment of invertebrate biodiversity on rocky shores: where there's a whelk there's way. Biodivers. Conserv. 14: 3565-3576 http://dx.doi.org/10.1007/s10531-004-0828-3

Somoza L., Medialdea T., León R., et al. 2012. Structure of mud volcano systems and pockmarks in the region of the Ceuta Contourite Depositional System (Western Alborán Sea). Mar. Geol. 332-334: 4-26. http://dx.doi.org/10.1016/j.margeo.2012.06.002

Streftaris N., Zenetos A. 2007. Molluscan diversity in the N. East Aegean - Greece. Rapp. Comm. Int. Mer Médit., 38: 607.

Templado J., Calvo M. Moreno D. et al. 2006. Flora y Fauna de la Reserva Marina y Reserva de Pesca de la isla de Alborán. Secretaría General de Pesca Marítima (Ministerio de Agricultura, Pesca y Alimentación), Museo Nacional de Ciencias Naturales (Consejo Superior de Investigaciones Científicas), Madrid, $269 \mathrm{pp}$.

UNEP-MAP-RAC/SPA. 2010a. Overview of scientific findings and criteria relevant to identifying SPAMIs in the Mediterranean open seas, including the deep sea (Notarbartolo di Sciara, G. and Agardy, T. eds) RAC/SPA, Tunis, $71 \mathrm{pp}$.

UNEP-MAP-RAC/SPA. 2010b. Technical report on the geographical information system developed for Mediterranean open seas (Requena S., Ed.) RAC/SPA, Tunis, 50 pp.

Warén A. 1984. A generic revision of the family Eulimidae (Gastropoda, Prosobranchia). J. Moll. Stud. Suppl. 13: 1-96.

WoRMS Editorial Board 2014. World Register of Marine Species. Available from <http://www.marinespecies.org> at Vlaams Instituut voor de Zee. 